\title{
Stochastic uncertainty analysis for unconfined flow systems
}

\author{
Gaisheng Liu, ${ }^{1,2}$ Dongxiao Zhang, ${ }^{1,3}$ and Zhiming $\mathrm{Lu}^{4}$ \\ Received 27 November 2005; revised 11 April 2006; accepted 5 June 2006; published 19 September 2006.
}

[1] A new stochastic approach proposed by Zhang and Lu (2004), called the

Karhunen-Loeve decomposition-based moment equation (KLME), has been extended to solving nonlinear, unconfined flow problems in randomly heterogeneous aquifers. This approach is on the basis of an innovative combination of Karhunen-Loeve decomposition, polynomial expansion, and perturbation methods. The random logtransformed hydraulic conductivity field $\left(\ln K_{S}\right)$ is first expanded into a series in terms of orthogonal Gaussian standard random variables with their coefficients obtained as the eigenvalues and eigenfunctions of the covariance function of $\ln K_{S}$. Next, head $h$ is decomposed as a perturbation expansion series $\Sigma h^{(m)}$, where $h^{(m)}$ represents the $m$ th-order head term with respect to the standard deviation of $\ln K_{S}$. Then $h^{(m)}$ is further expanded into a polynomial series of $m$ products of orthogonal Gaussian standard random variables whose coefficients $h_{i_{1}, i_{2}}^{(m)}, \ldots, i_{m}$ are deterministic and solved sequentially from low to high expansion orders using MODFLOW-2000. Finally, the statistics of head and flux are computed using simple algebraic operations on $h_{i_{1}, i_{2}, \ldots, i_{m}}^{(m)}$. A series of numerical test results in 2-D and 3-D unconfined flow systems indicated that the KLME approach is effective in estimating the mean and (co)variance of both heads and fluxes and requires much less computational effort as compared to the traditional Monte Carlo simulation technique.

Citation: Liu, G., D. Zhang, and Z. Lu (2006), Stochastic uncertainty analysis for unconfined flow systems, Water Resour. Res., 42, W09412, doi:10.1029/2005WR004766.

\section{Introduction}

[2] It has been long recognized that geological media possess a significant amount of spatial variability in the lithologic, petrophysical and structural components such that their key medium properties, e.g., permeability and porosity, which are often measured at only a few locations, are subject to several sources of errors when extending the limited data to areas where measurements are not available. As a result, the equations that govern subsurface physical and chemical fluid processes become stochastic and model predictions are best described as probability distributions instead of deterministic quantities. To evaluate uncertainties in subsurface fluid modeling, stochastic approaches have been developed [Dagan, 1989; Gelhar, 1993; Cushman, 1997; Zhang, 2002; Rubin, 2003]. Although other types of uncertainties are possible [Zhang, 2002], only the parameter uncertainty of the permeability is examined in this work.

[3] On the basis of how uncertainty is treated, two different stochastic frameworks can be identified in a general sense, namely, Monte Carlo (MC) simulation and

\footnotetext{
${ }^{1}$ Mewbourne School of Petroleum and Geological Engineering, University of Oklahoma, Norman, Oklahoma, USA.

${ }^{2}$ Now at Kansas Geological Survey, University of Kansas, Lawrence, Kansas, USA.

${ }^{3}$ Department of Energy and Resources Engineering, College of Engineering, Peking University, Beijing, China.

${ }^{4}$ Hydrology and Geochemistry Group, Los Alamos National Laboratory, Los Alamos, New Mexico, USA.

Copyright 2006 by the American Geophysical Union. 0043-1397/06/2005WR004766
}

the moment equation (ME) approach. In the MC simulation method [e.g., Hassan et al., 1998; Ballio and Guadagnini, 2004], uncertainty is indirectly considered through multiple, equally probable realizations of uncertain medium parameters. Each realization is then evaluated individually in a deterministic manner and the statistics of model predictions are computed from the results of all the parameter realizations. The MC method is conceptually straightforward and is easy to apply in practice. The major disadvantage is that it generally requires an extensive, sometimes formidable, computational effort, especially when the system under consideration is large-scale and exhibits nonlinear mechanics. Diagnostic criteria are often not obvious for evaluating statistical convergence in MC simulations [Ballio and Guadagnini, 2004], which further hinders its applicability in practice. On the other hand, in the ME approach [Dagan, 1982, 1985; Graham and McLaughlin, 1989; Neuman, 1993; Deng and Cushman, 1995; Hsu et al., 1996; Ghanem, 1998; Zhang, 1998; Amir and Neuman, 2001; Zhang and Lu, 2002], uncertainty is directly incorporated into governing equations. Using the perturbation technique, equations are derived for the statistical moments (typically the first two moments, mean and covariance) of model predictions. Generally, analytical solutions of the moment equations are difficult to obtain except for some limiting cases under simplified conditions. The computational effort required in the numerical $\mathrm{ME}$ approach increases rapidly with the size of model, which makes its application infeasible in most field practices.

[4] In recent years new stochastic approaches have been developed that rely on the Karhunen-Loeve (KL) decom- 
position of the underlying random fields [Ghanem and Spanos, 1991; Ghanem and Dham, 1998; Zhang and Lu, 2004]. Ghanem and Spanos [1991] used the KarhunenLoeve (KL) decomposition [Loeve, 1977] to expand the independent random processes and the dependent variables were represented by an orthogonal basis of polynomial chaos. This KL/polynomial chaos method has been applied to developing a two-dimensional multiphase model in a random porous medium [Ghanem and Dham, 1998].

[5] Zhang and $\mathrm{Lu}$ [2004] proposed a new approach, called the Karhunen-Loeve decomposition-based moment equation (KLME), to calculate the mean and covariance of hydraulic heads where the permeability field is randomly heterogeneous and flow is saturated and confined. Instead of the polynomial chaos basis, dependent variables were expanded into a perturbation series in which each individual term was further decomposed as products of orthogonal random variables. The statistical moments of model predictions were constructed algebraically based on the coefficients of dependent variable expansions. Unlike the conventional ME method, it has been demonstrated that the KLME approach does not require solving the covariance equations directly and is thus much more efficient computationally. In contrast to the $\mathrm{KL} /$ polynomial chaos approach that leads to coupled equations, the coefficient equations in KLME can be solved sequentially from low to high expansion orders and are independent within the same order. Yang et al. [2004] applied the KLME approach to saturatedunsaturated single-phase flow. Lu and Zhang [2004b] extended the approach to efficiently incorporate existing direct measurements of the hydraulic conductivity. Chen et al. [2005] applied the approach to modeling steady state two-phase flow.

[6] In this work, we extend the KLME approach to unconfined flow systems. Despite the relative large body of literature on stochastic hydrology, little attention has been paid to uncertainty analysis on unconfined flow under general conditions. Compared to confined flow, the unconfined flow governing equation is generally formulated as nonlinear in groundwater simulation and such nonlinearity is the major factor that slows the progress of stochastic research on the subject.

[7] The purpose of this study is to investigate the effects of randomness in the hydraulic conductivity field upon the statistics of model predictions (i.e., hydraulic heads and Darcy fluxes). Our strategy is to use the KL decomposition technique to expand the random hydraulic field and the perturbation method to expand model predictions. The expanded equations are reformulated such that they share the same structure as the original governing equation and therefore any existing simulator, such as MODFLOW-2000 [Harbaugh et al., 2000], can be taken advantage of to speed up the solution procedure.

[8] The remainder of this paper is organized as follows. We start by introducing the mathematical model that we solve for unconfined flow in this work. In line with the MODFLOW-2000 formulation, the governing equations for a general 3-D unconfined system are obtained by modifying the general Boussinesq equation for 2-D unconfined flow and taking into account vertical flow between top unconfined layer and underlying confined portions. Next, we derive a system of stochastic flow equations based on the
KLME approach and describe their solution procedure. Finally, we evaluate the accuracy and computational efficiency of the KLME approach in unconfined flow systems as compared to the traditional MC simulation technique and offer some concluding remarks summarized from this work.

\section{Stochastic Formulation of Unconfined Flow}

\subsection{Mathematical Model}

[9] The 2-D unconfined flow governing equation under the Dupuit assumption can be written as [Bear, 1972],

$$
\nabla_{x y} \cdot\left[K_{S}(\boldsymbol{x}) h_{B}(\boldsymbol{x}, t) \nabla_{x y} h(\boldsymbol{x}, t)\right]+g_{u}=S_{y} \frac{\partial h(\boldsymbol{x}, t)}{\partial t}
$$

where $K_{S}(\boldsymbol{x})$ is the hydraulic conductivity; $h_{B}(\boldsymbol{x}, t)=$ $h(\boldsymbol{x}, t)-B O T 1$ is the saturated thickness, $h(\boldsymbol{x}, t)$ is the hydraulic head; BOT1 is the bottom elevation of the unconfined aquifer; $g_{u}(\mathrm{~L} / \mathrm{T})$ is the sink/source term applied to the water table layer (e.g., recharge); $S_{y}$ is specific yield; $\boldsymbol{x}=$ $(x, y)^{T}$ is the spatial Cartesian coordinate vector (where superscript $T$ indicates transpose); $t$ is time; and $\nabla_{x y}$ is defined as $(\partial / \partial x, \partial / \partial y)$. Note that when $B O T 1$ is assumed to be zero, $h_{B}(\boldsymbol{x}, t)$ reduces to $h(\boldsymbol{x}, t)$.

[10] In practice, however, it is generally more accurate to represent the unconfined flow system as several different layers in 3-D, with the top layer often under water table conditions. Water communicates vertically between the top water table layer and underlying confined portions. Taking into account the vertical flow between water table and confined layers, we write the governing equation for the top water table layer as

$$
\begin{aligned}
\nabla_{x y} \cdot & {\left[K_{S}(\boldsymbol{x}) h_{B}(\boldsymbol{x}, t) \nabla_{x y} h(\boldsymbol{x}, t)\right]+K_{S}(\boldsymbol{x}) \frac{\partial h(\boldsymbol{x}, t)}{\partial z} } \\
& +g_{u}=S_{y} \frac{\partial h(\boldsymbol{x}, t)}{\partial t}
\end{aligned}
$$

where $K_{S}(\boldsymbol{x}) \partial h(\boldsymbol{x}, t) / \partial z$ represents a Darcy's flux between the water table and underlying confined layers in the vertical direction. For the underlying confined portions, the governing equation can be written as [Bear, 1972]

$$
\nabla \cdot\left[K_{S}(\boldsymbol{x}) \nabla h(\boldsymbol{x}, t)\right]+g_{c}=S_{S} \frac{\partial h(\boldsymbol{x}, t)}{\partial t},
$$

where $g_{c}(1 / \mathrm{T})$ is the sink/source that is applied to the confined portions; $S_{S}$ is the specific storage; and $\nabla$ stands for $(\partial / \partial x, \partial / \partial y, \partial / \partial z)$. As shown above, equations (2a) and (2b) are coupled through vertical Darcy's flux.

[11] The coupled equations of the water table layer and the underlying confined portions are subject to following initial and boundary conditions:

$$
\begin{gathered}
h(\boldsymbol{x}, 0)=H_{0}(\boldsymbol{x}), \quad \boldsymbol{x} \in D, \\
h(\boldsymbol{x}, t)=H(\boldsymbol{x}, t), \quad \boldsymbol{x} \in \Gamma_{D}, \\
K_{S}(\boldsymbol{x}) \nabla h(\boldsymbol{x}, t) \cdot n(\boldsymbol{x})=-Q(\boldsymbol{x}, t), \quad x \in \Gamma_{N} .
\end{gathered}
$$


where $H_{0}(\boldsymbol{x})$ is the initial hydraulic head in the flow domain $D ; H(\boldsymbol{x}, t)$ is the specified head on the Dirichlet boundary segments $\Gamma_{\mathrm{D}} ; Q(\boldsymbol{x}, t)$ is the flux across Neumann boundary segments $\Gamma_{\mathrm{N}}$; and $\boldsymbol{n}(\boldsymbol{x})$ is an outward unit vector normal to the boundary $\Gamma_{D} \cup \Gamma_{N}$. Note that equations (2a)-(2e) are in accordance with the mathematical formulation solved by the U.S. Geological Survey model MODFLOW-2000 [Harbaugh et al., 2000] in unconfined situations.

[12] In this study, $K_{S}(\boldsymbol{x})$ is taken as a random function and (2a)-(2e) become stochastic partial differential equations. All other parts of the flow model, i.e., sink/source, storage parameters and initial/boundary conditions, are assumed to be deterministic. Our goal here is to solve for the mean head and its associated uncertainty (expressed in terms of variance). The flux statistics can also be estimated after the stochastic flow equations are solved [Lu and Zhang, 2004a].

[13] As mentioned earlier, to solve stochastic equations (2a)-(2e), we apply the KLME approach proposed by Zhang and $\mathrm{Lu}$ [2004]. In this approach, the log-transformed hydraulic conductivity field is first expanded into a series in terms of orthogonal random variables using KL decomposition. Next, head is expanded into a perturbation series in which each individual term was further decomposed as products of orthogonal random variables. Finally, the statistical moments of model predictions (i.e., heads and fluxes) are constructed algebraically based on the coefficients of dependent variable expansions. All equations for these coefficients are reformulated such that they share exactly the same structure with original flow equation, which allows us to take advantage of any existing groundwater simulator, such as MODFLOW-2000, as the solver.

\subsection{Karhunen-Loeve Expansion of Hydraulic Conductivity}

[14] Let $Y(\boldsymbol{x})$ be the natural logarithm of a hydraulic conductivity field, $Y(\boldsymbol{x})=\ln \left[K_{S}(\boldsymbol{x})\right]$. Its covariance function $C_{Y}\left(\boldsymbol{x}_{1}, \boldsymbol{x}_{2}\right)=\left\langle Y^{\prime}\left(\boldsymbol{x}_{1}\right) Y^{\prime}\left(\boldsymbol{x}_{2}\right)\right\rangle$, where the perturbation $Y^{\prime}(\boldsymbol{x})$ is defined as $Y^{\prime}(\boldsymbol{x})=Y(\boldsymbol{x})-\langle Y(\boldsymbol{x})\rangle$ and \langle\rangle represents the mean (expectation) operator, is positive definite. The hydraulic conductivity $K_{S}(\boldsymbol{x})$ can be written as

$$
K_{S}(\boldsymbol{x})=\exp [Y(\boldsymbol{x})]=K_{G}(\boldsymbol{x}) \sum_{m=0}^{\infty} \frac{\left[Y^{\prime}(\boldsymbol{x})\right]^{m}}{m !}
$$

where $K_{G}(\boldsymbol{x})=\exp [\langle Y(\boldsymbol{x})\rangle]$ is the geometric mean of hydraulic conductivity $K_{S}(\boldsymbol{x})$, which represents a relatively smooth unbiased estimate of the unknown random function $K_{S}(\boldsymbol{x})$ and can be estimated using geostatistical approaches, such as kriging methods.

[15] Since the covariance function of $Y(\boldsymbol{x})$ is positive definite, it can be decomposed as [Courant and Hilbert, 1953]

$$
C_{Y}\left(\boldsymbol{x}_{1}, \boldsymbol{x}_{2}\right)=\sum_{n=1}^{\infty} \lambda_{n} \varphi_{n}\left(\boldsymbol{x}_{1}\right) \varphi_{n}\left(x_{2}\right)
$$

where $\lambda_{n}$ and $\varphi_{n}(\boldsymbol{x})$ are respectively the eigenvalues and deterministic eigenfunctions that can be determined by solving the following Fredholm equation analytically or numerically [Courant and Hilbert, 1953]:

$$
\int_{D} C_{Y}\left(\boldsymbol{x}_{1}, \boldsymbol{x}_{2}\right) \varphi\left(\boldsymbol{x}_{1}\right) d \boldsymbol{x}_{1}=\lambda \varphi\left(\boldsymbol{x}_{2}\right) .
$$

For some special covariance functions, $\lambda_{n}$ and $\varphi_{n}(\boldsymbol{x})$ can be solved analytically [Zhang and Lu, 2004]. Numerical solutions of eigenvalues and eigenfunctions are available [Press et al., 1992]. The set of eigenvalues $\lambda_{n}$ can be arranged as a nonincreasing series with respect to subscript $n$ and the rate of decrease depends on the ratio of the correlation length of the random conductivity field to the size of physical domain [Ghanem and Dham, 1998; Zhang and $L u, 2004]$. The effects of correlation lengths on the stochastic solutions of (2) and on the performance of the proposed KLME approach will be discussed in section 3 .

[16] The mean-removed stochastic process $Y^{\prime}(\boldsymbol{x})$ can be expanded in terms of $\lambda_{n}$ and $\varphi_{n}(\boldsymbol{x})$ [Loeve, 1977],

$$
Y^{\prime}(\boldsymbol{x})=\sum_{n=1}^{\infty} \xi_{n} \sqrt{\lambda_{n}} \varphi_{n}(\boldsymbol{x})
$$

where $\xi_{n}$ are the orthogonal random variables satisfying $\left\langle\xi_{n}\right\rangle=0$ and $\left\langle\xi_{m} \xi_{n}\right\rangle=\delta_{m n}, \delta_{m n}$ taking value 1 when $m=n$ and 0 otherwise. Equation (6a) is called the KL expansion, which can be interpreted as a decomposition of stochastic process $Y(\boldsymbol{x})$ in a space spanned by the set of orthogonal random variables $\xi_{n}$. These random variables are Gaussian if $Y(\boldsymbol{x})$ is Gaussian. Because the eigenvalue $\lambda_{n}$ and the corresponding eigenfunction $\varphi_{n}(\boldsymbol{x})$ always appear together, we combine them into a new function $f_{n}(\boldsymbol{x})=\sqrt{\lambda_{n}} \varphi_{n}(\boldsymbol{x})$,

$$
Y^{\prime}(\boldsymbol{x})=\sum_{n=1}^{\infty} \xi_{n} f_{n}(\boldsymbol{x})
$$

\subsection{Karhunen-Loeve Expansion-Based Moment Equations (KLME)}

[17] In the KLME approach, as is done in the conventional ME method, hydraulic head is first decomposed with a perturbation expansion as,

$$
h(\boldsymbol{x}, t)=\sum_{m=0}^{\infty} h^{(m)}(\boldsymbol{x}, t)
$$

where $h^{(m)}(\boldsymbol{x}, t)$ is the $m$ th-order expansion in terms of the standard deviation of $\log$ hydraulic conductivity, $\sigma_{Y}$. The term $h_{B}(\boldsymbol{x}, t)$ is expanded similarly such that $h_{B}^{(0)}(\boldsymbol{x}, t)=$ $h^{(0)}(\boldsymbol{x}, t)-B O T 1$ and $h_{B}^{(i)}(\boldsymbol{x}, t)=h^{(i)}(\boldsymbol{x}, t)$ for $i \geq 1$. Substituting expansions of $h^{(m)}(\boldsymbol{x}, t), h_{B}(\boldsymbol{x}, t)$, and $K_{S}(\boldsymbol{x})$ into equations (1) and (2) and separating terms at different orders, one obtains a set of expanded equations for different orders of head terms. Dropping coordinate $\boldsymbol{x}$ and time $t$ for simplicity, the zeroth-order equations for the top water table layer and the underlying confined layers can be written respectively as

$$
\nabla_{x y} \cdot\left[K_{G} h_{B}^{(0)} \nabla_{x y} h^{(0)}\right]+g_{u}+K_{G} \frac{\partial h^{(0)}}{\partial z}=S_{y} \frac{\partial h^{(0)}}{\partial t},
$$




$$
\nabla \cdot\left[K_{G} \nabla h^{(0)}\right]+g_{c}=S_{S} \frac{\partial h^{(0)}}{\partial t}
$$

subject to initial and boundary conditions

$$
\begin{gathered}
h^{(0)}(\boldsymbol{x}, 0)=H_{0}(\boldsymbol{x}), \quad \boldsymbol{x} \in D, \\
h^{(0)}(\boldsymbol{x}, t)=H(\boldsymbol{x}, t), \quad \boldsymbol{x} \in \Gamma_{D}, \\
K_{G} \nabla h^{(0)}(\boldsymbol{x}, t) \cdot \boldsymbol{n}(\boldsymbol{x})=-Q(\boldsymbol{x}, t), \quad \boldsymbol{x} \in \Gamma_{N} .
\end{gathered}
$$

The first-order equations for the top water table layer and the underlying confined layers can be written respectively as

$$
\begin{gathered}
\nabla_{x y} \cdot\left[K_{G} h_{B}^{(0)} \nabla_{x y} h^{(1)}\right]+K_{G} \frac{\partial h^{(1)}}{\partial z}+g_{u}^{(1)}=S_{y} \frac{\partial h^{(1)}}{\partial t}, \\
\nabla \cdot\left[K_{G} \nabla h^{(1)}\right]+g_{c}^{(1)}=S_{S} \frac{\partial h^{(1)}}{\partial t},
\end{gathered}
$$

subject to

$$
\begin{aligned}
h^{(1)}(\boldsymbol{x}, 0) & =0, \quad \boldsymbol{x} \in D, \\
h^{(1)}(\boldsymbol{x}, t) & =0, \quad \boldsymbol{x} \in \Gamma_{D}, \\
K_{G} \nabla h^{(1)}(\boldsymbol{x}, t) \cdot \boldsymbol{n}(\boldsymbol{x}) & =Y^{\prime} Q(\boldsymbol{x}, t), \quad \boldsymbol{x} \in \Gamma_{N},
\end{aligned}
$$

where

$$
\begin{gathered}
g_{u}^{(1)}=\nabla_{x y} \cdot\left[K_{G}\left(Y^{\prime} h_{B}^{(0)}+h^{(1)}\right) \nabla_{x y} h^{(0)}\right]+K_{G} Y^{\prime} \frac{\partial h^{(0)}}{\partial z}, \\
g_{c}^{(1)}=\nabla \cdot\left[K_{G} Y^{\prime} \nabla h^{(0)}\right] .
\end{gathered}
$$

Similarly one can derive equations for higher-order head terms (see Appendix A). In the conventional ME approach, head statistics are directly solved from moment equations that are derived from these equations. For instances, taking ensemble mean of equations (A1)-(A7) yields the equations with initial and boundary conditions for the secondorder correction, $\left\langle h^{(2)}\right\rangle$. The equations for the first-order head covariance $C_{h}(\boldsymbol{x}, t ; \boldsymbol{y}, \tau)$ can be obtained by multiplying equations $(9 \mathrm{a})-(9 \mathrm{~g})$ by $h^{(1)}(\boldsymbol{y}, \tau)$ and then taking ensemble mean. Owing to the large dimensionality of covariance equations, the conventional ME method can be computationally very demanding for large-scale problems. It has been demonstrated that corrections of second- and higher-order are extremely difficult to implement in the conventional ME method except under certain simplified conditions [Hsu et al., 1996; Zhang, 2002].
[18] In the KLME method, however, one does not solve higher-order moment equations directly. Instead, $h^{(m)}$ are further expanded in terms of the orthogonal random variables that are used to decompose $Y^{\prime}(\boldsymbol{x})$,

$$
h^{(m)}=\sum_{i_{1}, i_{2}, \ldots, i_{m}=1}^{\infty}\left(\prod_{j=1}^{m} \xi_{i_{j}}\right) h_{i_{1}, i_{2}, \ldots, i_{m}}^{(m)}
$$

where $h_{i_{1}, i_{2}}^{(m)}, \ldots, i_{m}$ are deterministic functions to be determined; $i_{1}, i_{2}, \ldots, i_{m}$ are referred to as modes of head at the $m$ th order. It is noteworthy that the polynomial chaos expansions by Ghanem and Spanos [1991] are not suitable for expanding $h^{(m)}$ individually. For instance, if $h^{(2)}$ is expanded using the second-order polynomial chaos $\left\{\xi_{i_{1}} \xi_{i_{2}}-\delta_{i_{1} i_{2}}\right\}$ as $h^{(2)}=\sum_{i_{1}, i_{2}}\left(\xi_{i_{1}} \xi_{i_{2}}-\delta_{i_{1} i_{2}}\right) h_{i_{1} i_{2}}^{(2)}$, this will result in $\left\langle h^{(2)}\right\rangle^{\left(i_{1} i_{2}\right.}=0$ as $\left\langle\xi_{i_{1}} \xi_{i_{2}}-\delta_{i_{1} i_{2}}\right\rangle \equiv 0$. However, in general, $\left\langle h^{(2)}\right\rangle$ is not equal to zero unless the medium is unbounded and statistically homogeneous [Zhang, 2002].

[19] By substituting (10) and the KL decomposition of $Y^{\prime}(\boldsymbol{x})$, i.e., (6b), into (9) and recalling the fact that the set $\left\{\xi_{i}\right\}$ are orthogonal, one obtains the governing equations for the first-order mode coefficients $h_{i}^{(1)}$ for the top water table layer and the underlying confined portions,

$$
\nabla_{x y} \cdot\left[K_{G} h_{B}^{(0)} \nabla_{x y} h_{i}^{(1)}\right]+K_{G} \frac{\partial h_{i}^{(1)}}{\partial z}+g_{u_{i}}^{(1)}=S_{y} \frac{\partial h_{i}^{(1)}}{\partial t},
$$

$$
\nabla \cdot\left[K_{G} \nabla h_{i}^{(1)}\right]+g_{c_{i}}^{(1)}=S_{S} \frac{\partial h_{i}^{(1)}}{\partial t}
$$

subject to initial and boundary conditions

$$
h_{i}^{(1)}(\boldsymbol{x}, 0)=0, \quad \boldsymbol{x} \in D,
$$

$$
h_{i}^{(1)}(\boldsymbol{x}, t)=0, \quad \boldsymbol{x} \in \Gamma_{D},
$$

$$
K_{G} \nabla h_{i}^{(1)}(\boldsymbol{x}, t) \cdot \boldsymbol{n}(\boldsymbol{x})=f_{i} Q(\boldsymbol{x}, t), \quad \boldsymbol{x} \in \Gamma_{N},
$$

where

$$
\begin{gathered}
g_{u_{i}}^{(1)}=\nabla_{x y} \cdot\left\{K_{G}\left[f_{i} h_{B}^{(0)}+h_{i}^{(1)}\right] \nabla_{x y} h^{(0)}\right\}+K_{G} f_{i} \frac{\partial h^{(0)}}{\partial z}, \\
g_{c_{i}}^{(1)}=\nabla \cdot\left[K_{G} f_{i} \nabla h^{(0)}\right] .
\end{gathered}
$$

Similarly one can derive equations for higher-order mode coefficients $h_{i_{1}, i_{2}, \ldots, i_{m}}^{(m)}$ at $m \geq 2$ (refer to Appendix B).

[20] Comparing the equations between the water table layer (e.g., (11a)) and underlying confined portions (e.g., (11b)), it is clear that the nonlinearity resulting from unconfined flow adds a large complexity to the KLME formulations. The artificial source/sink terms in (11a) and (11b) contain more terms for the water table layer than those for the underlying confined portions. Moreover, the coefficients to be solved, i.e., $h_{i}^{(1)}$, appear in (11f), which means 


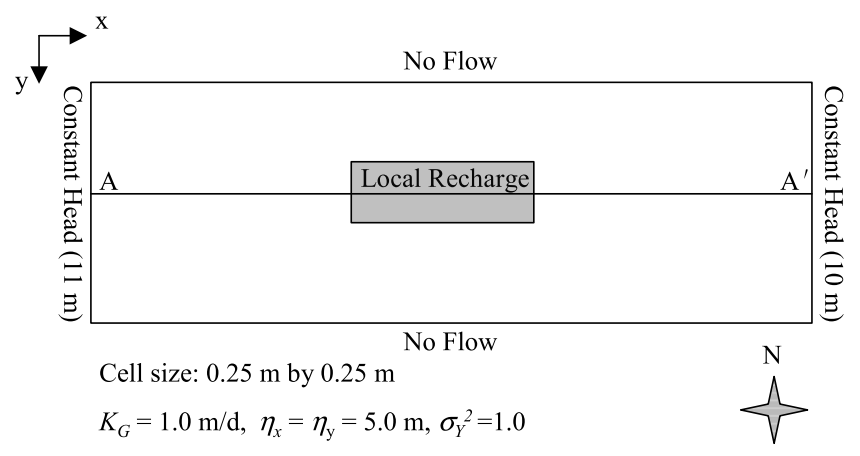

Figure 1. Schematic diagram of model setup in the 2-D unconfined flow problem. Line A-A' indicates the location along which results are compared between the KLME and $\mathrm{MC}$ approaches.

that equations (11) are nonlinear and must be solved in an iterative manner whenever unconfined flow is present.

[21] Once the deterministic coefficients $h_{i_{1}, i_{2}}^{(m)} \ldots, i_{m}$ are calculated, one can easily compute the mean head and its variance (as well as other moments) by simple algebraic operations. For example, up to the third order in $\sigma_{Y}, h \approx$ $\sum_{m=0}^{3} h^{(m)}$, the mean head can be approximated as

$$
\langle h\rangle \approx \sum_{m=0}^{3}\left\langle h^{(m)}\right\rangle=h^{(0)}+\sum_{i=1}^{\infty} h_{i i}^{(2)},
$$

where the first term in the right-hand side is the zeroth-order mean head solution and the second term represents the second-order correction, where terms $h_{i, j}^{(2)}$ for $i \neq j$ disappear because of $\left\langle\xi_{i} \xi_{j}\right\rangle=0$. The first-order correction to the zeroth-order mean is zero. The head variance can be approximated as

$$
\sigma_{h}^{2} \approx \sum_{i=1}^{\infty}\left[h_{i}^{(1)}\right]^{2}+2 \sum_{i, j=1}^{\infty}\left[h_{i, j}^{(2)}\right]^{2}+6 \sum_{i, j=1}^{\infty}\left[h_{i}^{(1)} h_{i, j, j}^{(3)}\right]
$$

where the first term in the right-hand side is the head variance up to the first order in the variance of $\log$ conductivity $\sigma_{Y}^{2}$; and the second and third terms represent the second-order correction in $\sigma_{Y}^{2}$. Detailed derivations of (12) and (13) are given by Zhang and Lu [2004]. The mean and variance of fluxes are computed in a similar manner [Lu and Zhang, 2004a].

[22] Note that equations for $h_{i, i}^{(m)}, \ldots, i$, as shown in (8) and (11) for $h^{(0)}$ and $h_{i}^{(1)}$, have been formulated in the same structure as the original flow equations (2a)-(2e). Therefore any existing simulator, such as MODFLOW2000 [Harbaugh et al., 2000], can be directly used to solve $h_{i_{1}, i_{2}, \ldots, i_{m}}^{(m)}$. Moreover, due to the same structure of these equations, the left-hand side coefficient matrix remains unchanged for all numerical solutions of terms $h_{i_{1}, i_{2}, \ldots, i_{m}}^{(m)}$. This feature further allows us to significantly increase the computational efficiency of the KLME approach, which has been a major effort in one of our ongoing research projects.

[23] Equations for $h_{i_{1}, i_{2}, \ldots, i_{m}}^{(m)}$ can be solved sequentially from low to high order, and at the same order $m$ the equations are independent of each other, which means that equations at the same order can be solved in parallel. The solution process can be summarized as follows: (1) at the current time step, solving the zeroth-order term $h^{(0)}$ from (8), (2) solving $h_{i}^{(1)}$ from (11) for different modes $i$, (3) solving higher-order terms $h_{i j}^{(2)}, h_{i j k}^{(3)}$, etc., if needed, (4) computing the mean and variance of head using (12) and (13), and (5) adding a time increment and repeating steps $1-5$, if needed. A new code, MODFLOW-STO, has been developed to numerically implement the stochastic formulation presented in this study, in which MODFLOW-2000 is taken as a subroutine to solve for $h^{(0)}$ and $h_{i_{1}, i_{2}, \ldots, i_{m}}^{(m)}$.

\section{Illustrative Examples}

[24] Two hypothetical numerical experiments were conducted to examine the accuracy of the approach presented in this work for stochastic modeling of flow under unconfined conditions. Results from the KLME method were compared to those from the classical MC simulations. Computational efficiency was also compared between the KLME approach and $\mathrm{MC}$ simulations. To facilitate comparisons, identical numerical meshes were used in both approaches, although the KLME method may take a much coarser grid resolution. In following test problems the log conductivity field is assumed to be second-order stationary and follows a separable exponential covariance function,

$$
C_{Y}\left(\boldsymbol{x}_{1}, \boldsymbol{x}_{2}\right)=\sigma_{Y}^{2} \exp \left[-\frac{\left|x_{1}-x_{2}\right|}{\eta_{x}}-\frac{\left|y_{1}-y_{2}\right|}{\eta_{y}}-\frac{\left|z_{1}-z_{2}\right|}{\eta_{z}}\right],
$$

where $\eta_{x}, \eta_{y}$ and $\eta_{z}$ are the correlation lengths at $x, y$, and $z$ directions, respectively. Analytical solutions of eigenvalues and eigenfunctions are available for this covariance function [e.g., Zhang and Lu, 2004]. The equation is in threedimensional (3-D) form and can be reduced to 2-D $(x, y)$ plane by simply disregarding the $z$ component. Other types of covariance functions have been implemented in the code "MODFLOW-STO", in which the solutions of eigenvalues and eigenfunctions are obtained numerically [Press et al., 1992]. Preliminary investigations indicated that although the detailed results vary, general observations obtained from these covariance functions are similar to those with (14) regarding the KLME approach and are therefore not shown here.

\subsection{Two-Dimensional Unconfined Flow}

[25] In this example we tested the KLME approach in a 2 -D unconfined flow system. The flow domain is a rectangle of $30 \mathrm{~m}$ long by $10 \mathrm{~m}$ wide (Figure 1). There is no flow across the northern and southern boundaries, while on the west and east borders the heads are constant at $11 \mathrm{~m}$ and $10 \mathrm{~m}$, respectively. A finite difference mesh of 121 by 40 regular cells $(0.25 \mathrm{~m}$ on a side) is used to represent the domain. It is noteworthy that half of the constant head boundary cells are not actually involved in the block-centered calculation (which is the case in MODFLOW-2000); as a result one extra column of cells are added such that the effective size of the finite difference model is equal to that of the real problem. The origin of coordinate system is at the upper, left corner, and points to east in $x$ direction and to south in $y$ direction. Unless specified otherwise, the log conductivity 
(a) Mean Head

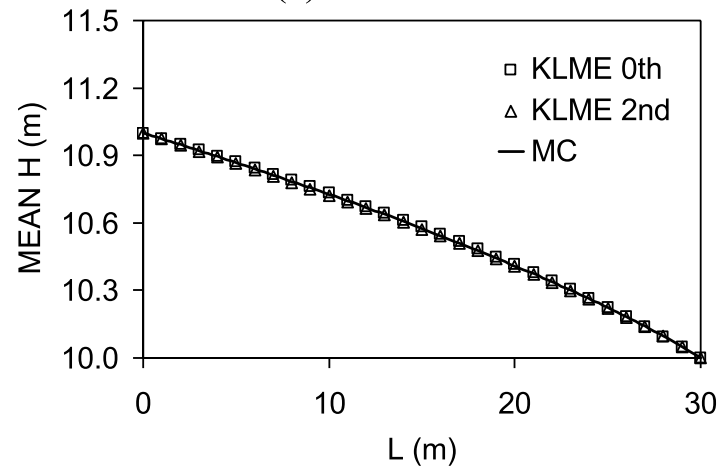

(c) Mean Flux

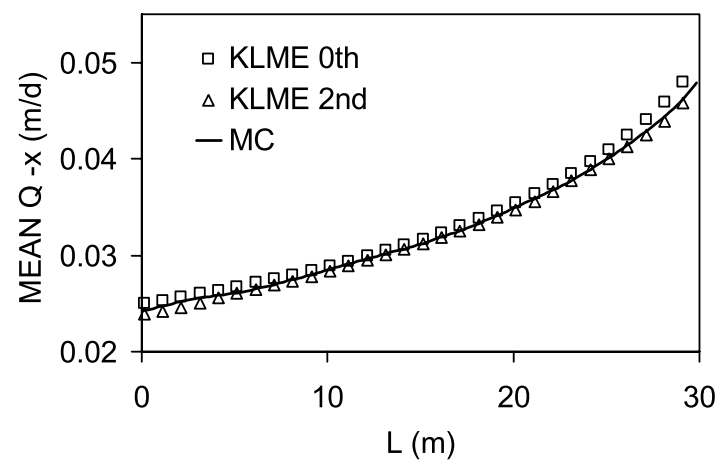

(b) Head Variance

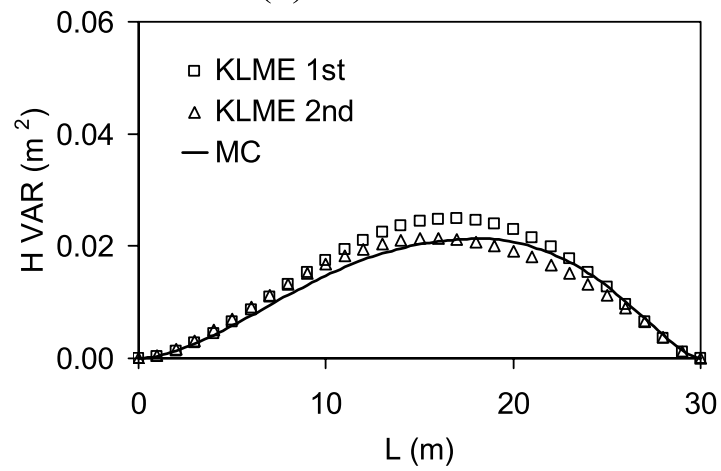

(d) Flux Variance

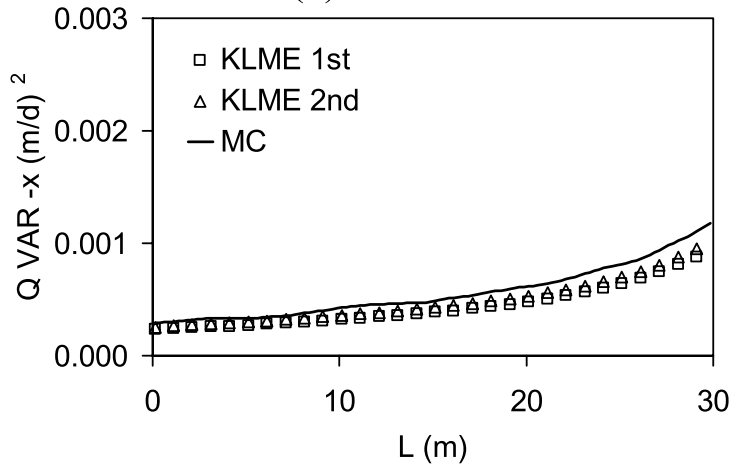

Figure 2. Mean and variance of heads and fluxes calculated along line A-A $\mathrm{A}^{\prime}$ in the 2-D steady state example without recharge: (a) mean head, (b) head variance, (c) mean flux, and (d) flux variance. The correlation lengths of random conductivity field are $5.0 \mathrm{~m}$ in both $x$ and $y$ directions, and the log variance is 1.0. For easy visualization the KLME results are plotted for one data point out of every four.

field has an unconditional zero mean $\left(K_{G}=1.0 \mathrm{~m} /\right.$ day $)$ variance $\sigma_{Y}^{2}=1.0$ and correlation lengths $\eta_{x}=\eta_{y}=5.0 \mathrm{~m}$.

[26] As mentioned earlier, in the KLME approach we obtained the solutions of $h_{i_{1}, i_{2}, \ldots, i_{m}}^{(m)}$ up to the third order, namely, $h^{(0)}, h_{i_{1}}^{(1)}, h_{i_{1}, i_{2}}^{(2)}$, and $h_{i_{1}, i_{2}, i_{3}}^{(3)}$. Unless specified otherwise, $h_{i_{1}}{ }^{(1)}$ were calculated for the first 100 modes, $i_{1}=\overline{1,100}$ at the first order; at the second order, $h_{i_{1}, i_{2}}^{(2)}$ were calculated for the first 20 by 20 modes, $i_{1}, i_{2}=\overline{1,20}$; and at the third order, $h_{i_{1}, i_{2}, i_{3}}^{(3)}$ were calculated for the first 10 by 10 by 10 modes, $i_{1}, i_{2}, i_{3}=\overline{1,10}$. Note that because of the symmetry of $h_{i_{1}, i_{2}, \ldots, i_{m}}^{(m)}$ with respect to their subscripts, we only needed to compute $h_{i_{1}, i_{2}}^{(2)}$ for $1 \leq i_{1} \leq i_{2} \leq 20$ and $h_{i_{1}, i_{2}, i_{3}}^{(3)}$ for $1 \leq i_{1} \leq i_{2} \leq i_{3} \leq 10$. Therefore a total of 1 (zeroth order $)+100$ (first order) +210 (second order) +220 (third order $)=531$ mode calculations are required at each time . These mode numbers were chosen sufficiently large that the statistics of $h_{i_{1}, i_{2}, \ldots, i_{m}}^{(m)}$ converge at the first three orders for different cases discussed below. In order to achieve both computational efficiency and accuracy, the optimal combination of mode numbers at different orders may vary from case to case, depending on the specific model setting and conductivity statistics under consideration. In the $\mathrm{MC}$ simulations it was found that generally about 5000 realizations were needed for the statistics of model outputs to converge in this example. The computational effort is approximately the same between one MC realization and one mode simulation.

\subsubsection{Steady State, No Recharge}

[27] In the first case we investigated a steady state flow field without recharge. Figures $2 \mathrm{a}$ and $2 \mathrm{~b}$ show the mean and variance of heads calculated from both KLME and MC approaches. Results are illustrated along a cross section A$\mathrm{A}^{\prime}$ (Figure 1) that runs through the middle of domain in the mean flow direction along which both head and flux quantities vary the most. For easy visualization, the KLME results are plotted for one data point out of every four. The zeroth-order mean head is based on the first term in the right-hand side of equation (12) only, while the secondorder mean includes both the first and second terms (i.e., the zeroth-order mean plus a second-order correction). Clearly, because of the nonlinear nature of flow, the head gradient is not uniform along the flow direction. The water table is relatively flat in the upstream region and becomes steeper in the downstream front. There is no distinguishable difference between all three mean heads due to the particular problem configuration in this case.

[28] Figure $2 \mathrm{~b}$ indicates that head variance is not symmetric in the unconfined flow system. Contrary to the results for confined flow [Zhang and $\mathrm{Lu}, 2004$ ], head variance in unconfined flow is asymmetric and skewed toward downstream where head gradient is larger. At the two constant head boundaries variance is zero as those heads are prescribed as constant and their values remain unchanged throughout the simulation. The first-order KLME head variance is based on the first term on the 
(a) Mean Head

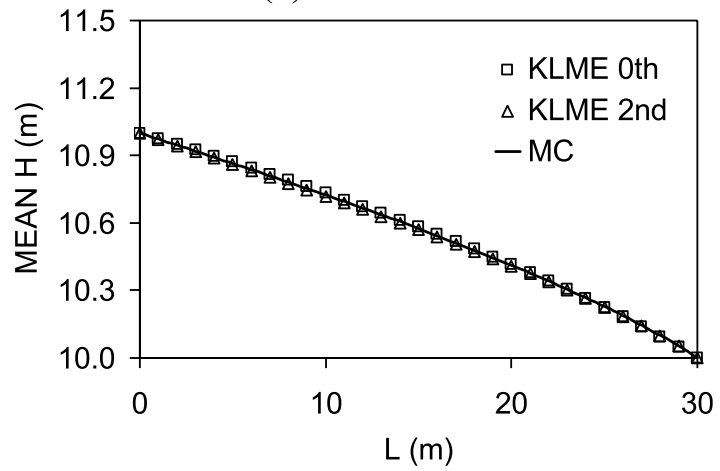

(c) Mean Flux

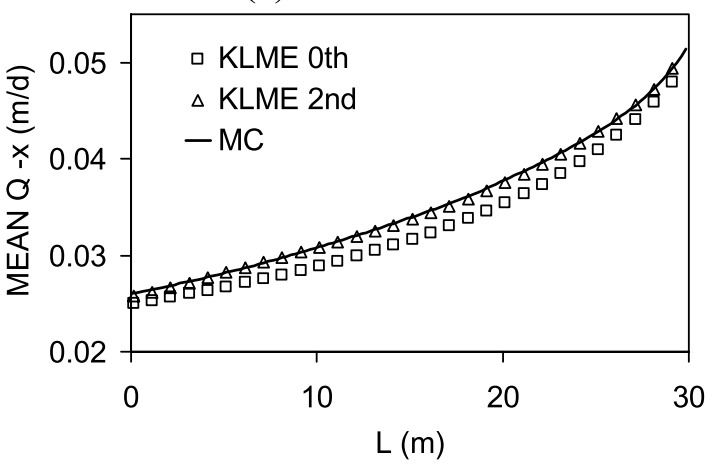

(b) Head Variance

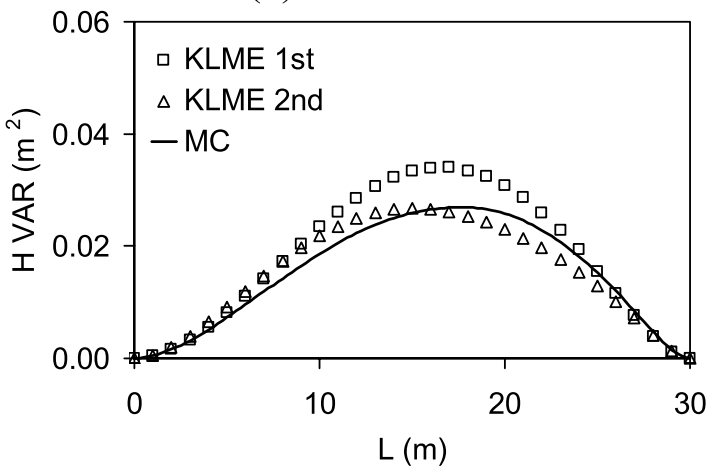

(d) Flux Variance

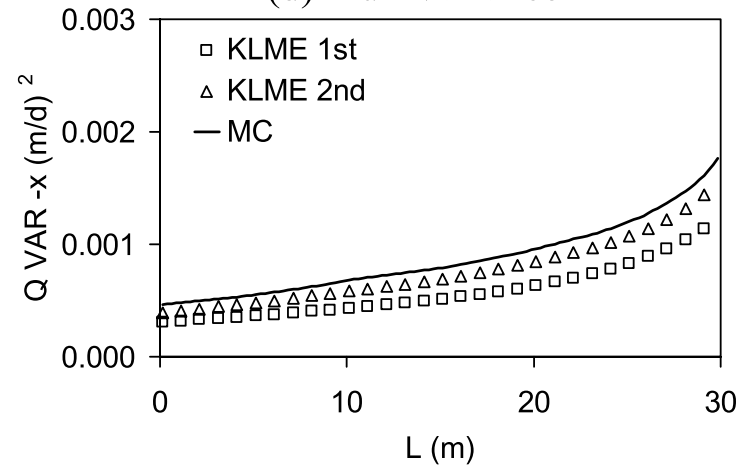

Figure 3. Mean and variance of heads and fluxes calculated along line $A-A^{\prime}$ when conductivity correlation lengths are increased to $10.0 \mathrm{~m}$ in both $x$ and $y$ directions: (a) Mean head, (b) head variance, (c) mean flux, and (d) flux variance. Other model settings remain identical to those for Figure 2.

right-hand side of (13), while the second-order variance includes both the first- and second-order contributions, i.e., all three terms of (13). Overall, both orders of head variance calculated from KLME are effective in representing the $\mathrm{MC}$ results. Close examination indicates that the calculated values of the first-order KLME head variance are generally larger than those of $\mathrm{MC}$, and the second-order correction is negative and improves solution accuracy by rectifying the overshoot in first-order calculation.

[29] The mean and variance of fluxes in the ambient flow direction $(x)$ are plotted in Figures $2 \mathrm{c}$ and $2 \mathrm{~d}$, respectively. Because of increasing head gradient, the mean value of fluxes increases in the downstream direction, and so does flux variance. The two KLME approximations of the mean fluxes are accurate, and the second-order correction shows slight improvement in matching the MC results, particularly in the downstream area. For flux variance, despite some underprediction, both orders of KLME values provide a consistently good approximation of the MC solution. Similar to the mean of fluxes, second-order flux variance shows slight improvement over the first-order result. It is noteworthy that because of the nonlinear nature of unconfined flow, the increase of both mean and variance of fluxes becomes accelerated in the flow direction.

[30] The effects of the correlation length and variance of the random conductivity field have been explored in detail by Zhang and Lu [2004] for confined flow systems. To investigate these effects for unconfined flow, we conducted two additional sets of numerical simulations here. First, the correlation lengths $\eta_{x}$ and $\eta_{y}$ are increased to $10.0 \mathrm{~m}$ while all other model settings remain unchanged. As demonstrated by Ghanem and Dham [1998] and Zhang and Lu [2004], larger correlation lengths increase the rate of decline in the series of eigenvalues $\lambda_{n}$ and intuitively, the number of modes required at each expansion order will be less. On the other hand, the contributions of higher-order terms to the overall solutions increase because the products of leading eigenvalues in the $\lambda_{n}$ series become relatively more significant. For examples, in the second-order mode equations (B6) and (B7) the artificial sink/source $g$ terms are proportional to the products of $f_{i}$ and $f_{j}, g_{u_{i, j}}^{(2)}, g_{c_{i, j}}^{(2)} \propto f_{i} f_{j}$, and in (B13) and (B14) $g_{u_{i, j, k}}^{(3)}, g_{c_{i, j, k}}^{(3)} \propto f_{i} f_{j} f_{k}$.

[31] Figure 3 shows the mean and variance of heads and fluxes computed from both MC and KLME approaches at correlation lengths $10.0 \mathrm{~m}$. There is no distinguishable change on mean heads. For the mean fluxes and variance of heads and fluxes, however, the computed statistics increase noticeably after correlation lengths are increased to $10.0 \mathrm{~m}$. Furthermore, the second-order correction in the KLME approach becomes more pronounced when correlation lengths increase, resulting in significantly better agreement between KLME and MC results for head variance, mean fluxes and flux variance. Comparisons between Figures 2 and 3 indicate that for small correlation lengths, the contributions of higher-order terms are relatively small and the first-order results alone provide an adequate approximation of the head and flux variance, although the number of modes required may be potentially 
(a) Mean Head

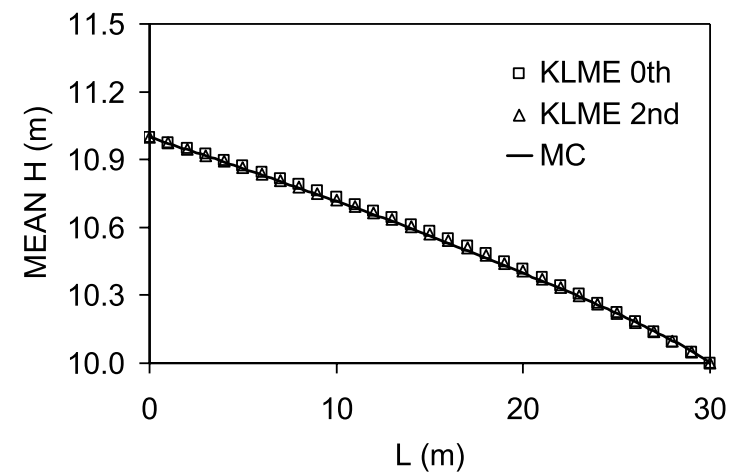

(c) Mean Flux

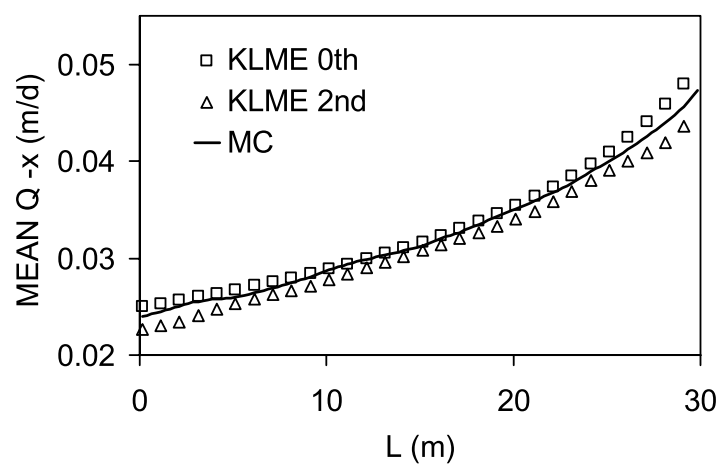

(b) Head Variance

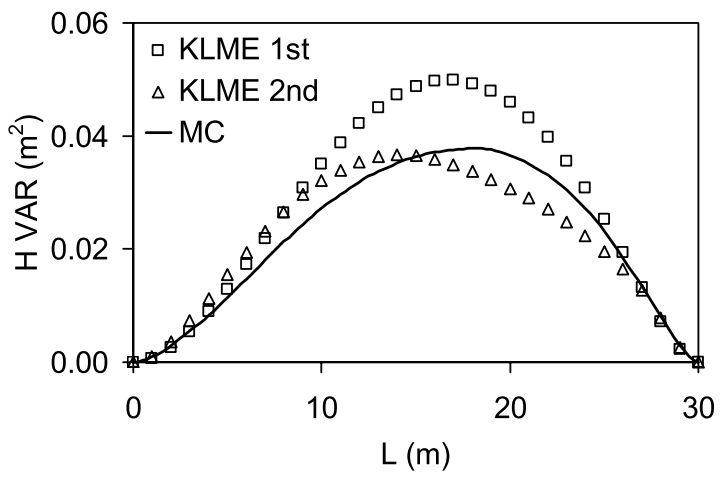

(d) Flux Variance

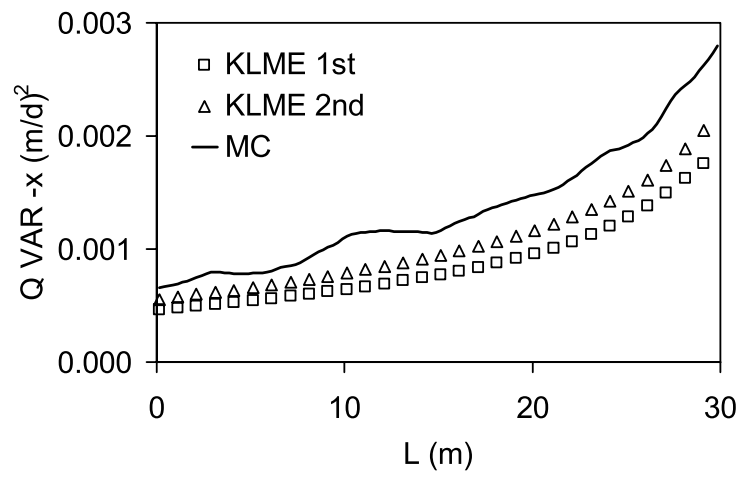

Figure 4. Mean and variance of heads and fluxes calculated along line $\mathrm{A}-\mathrm{A}^{\prime}$ when log conductivity variance is increased to 2.0: (a) mean head, (b) head variance, (c) mean flux, and (d) flux variance. Other model settings remain identical to those for Figure 2.

large. When correlation lengths become large, the higherorder terms become more important and need to be considered in order to better characterize flow statistics. However, the number of modes at each expansion order can be reduced. It is noteworthy that in this example the correlation lengths have been set extremely large as compared to the domain size, yet the second-order KLME results provide a very accurate match on both head and flux statistics such that the third or higher term is not required in the case of a moderate $\log$ conductivity variance.

[32] To investigate the effects of the variance of $\log$ conductivity, we increased $\sigma_{Y}^{2}$ from 1.0 to 2.0 while retained other model parameters identical to those for Figure 2. As demonstrated by Zhang and $L u$ [2004], the magnitudes of eigenvalues increase directly with the variance $\sigma_{Y}^{2}$ as it can be proved that $W \sigma_{Y}^{2}=\sum_{n=1}^{\infty} \lambda_{n}$, where $W$ is the measure of the domain size (the total length, area or volume for 1-D, 2-D, or 3-D domain, respectively). Intuitively, when $\sigma_{Y}^{2}$ is large, both higher-order terms and a larger number of modes at each expansion order are potentially needed in order to obtain accurate solutions. Figure 4 shows the results of head and $x$ flux moments when $\sigma_{Y}^{2}$ is increased to 2.0. Compared to Figure 2, the mean of heads and fluxes shows little change. The calculated variance for heads and fluxes, on the other hand, becomes substantially larger when the variance of underlying conductivity field increases. The first-order KLME head variance values are higher than those from $\mathrm{MC}$, and the second-order terms are capable of lowering the large overestimation back to the appropriate level. For flux variance, the first-order KLME results show a large underestimation and the second terms show more improvement in matching the MC solution than those in Figure 2 but not as much as those on head variance. The more mismatch error by KLME on flux statistics is likely due to the more nonlinear relationship between flux and conductivity (flux $q=K_{S} \nabla h$ where $h$ is further a function of $K_{S}$ ). It should be pointed out that when the $\log$ conductivity variance is increased to 2.0, the flux variances calculated by the MC approach also show some oscillations and additional realizations appear needed for better accuracy of MC results.

\subsubsection{Steady State With Recharge}

[33] Compared to the case in section 3.1.1, recharge is added in a region of 10 rows by 30 columns $(x: 11.125 \sim$ $18.625 \mathrm{~m} ; y: 3.75 \sim 6.25 \mathrm{~m}$ ) at rate $0.1 \mathrm{~m} / \mathrm{d}$. All other model settings remain unchanged. Figure 5 displays the calculated moments for heads and $x$ fluxes after recharge is included. In all situations the KLME approach is able to provide an effective agreement on $\mathrm{MC}$ solutions. It is seen that because of recharge, the mean heads are raised throughout the system and groundwater divide forms in the recharge area. The second-order correction shows some improvement over the zeroth-order mean heads in the KLME approach, especially in the vicinity of the recharge area. The calculated values for head variance are also raised by recharge. The head variance increases by about a factor of 3 
(a) Mean Head

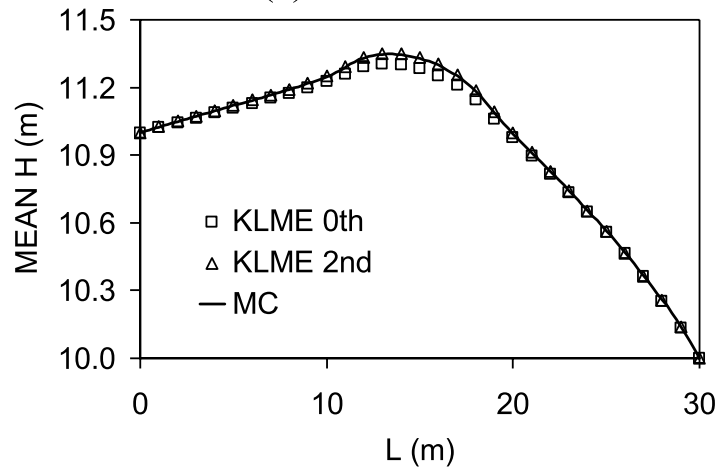

(c) Mean Flux

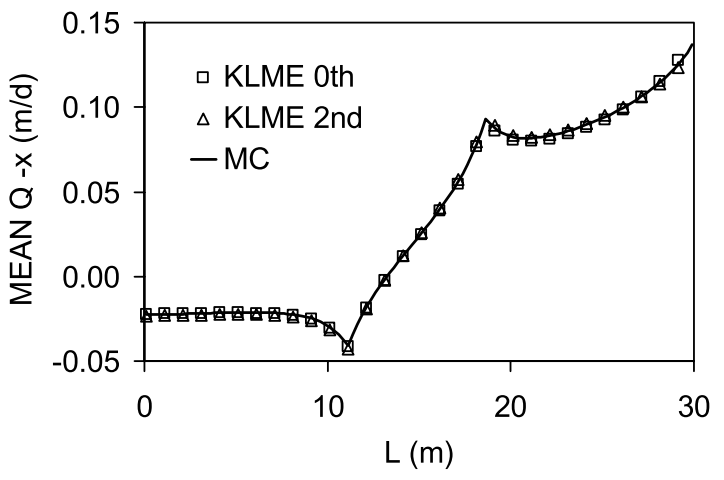

(b) Head Variance

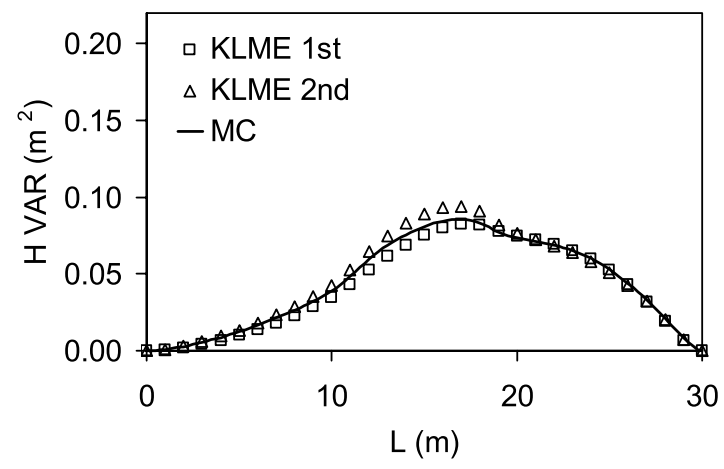

(d) Flux Variance

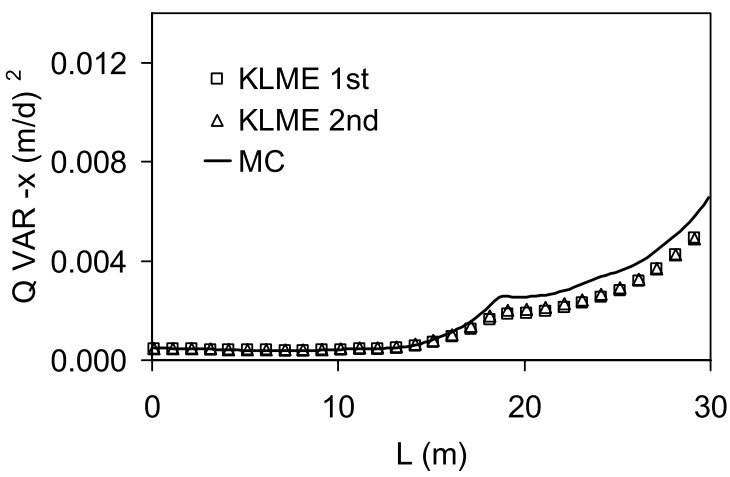

Figure 5. Mean and variance of heads and fluxes calculated along line $A-A^{\prime}$ in the 2-D steady state example with recharge: (a) mean head, (b) head variance, (c) mean flux, and (d) flux variance. Except for the added recharge, other model settings remain identical to those for Figure 2.

compared to Figure 2b. Furthermore, the increase in head variance is mostly in the downstream area and the resulting variance curves are more skewed. Unlike previous cases (Figures $2 b-4 b$ ), it is interesting to note that the first-order head variance calculated by KLME is smaller than the MC result when recharge is present. The second-order correction in KLME is positive and thus improves solution accuracy by rectifying the underprediction in first-order calculation. Consistent with the mean head distribution in Figure 5a, the mean flux becomes negative to the left side of rechargeinduced divide, indicating flow direction has been reversed toward the western boundary. Two local peaks are created on the mean flux profile by recharge. Figure $5 \mathrm{~d}$ shows that the variance of fluxes is significantly increased by recharge as compared to Figure 2d. Both orders of flux variance by KLME are effective in representing the MC result, and the second-order correction is minimal.

[34] Figures 6 and 7 present the calculated mean and variance of heads and $x$ fluxes, respectively, when the conductivity correlation lengths are increased to $10.0 \mathrm{~m}$ or $\log$ variance is increased to 2 . Other model settings remain identical to those for Figure 5. In both cases, the mean of heads and fluxes calculated by KLME is in good agreement with the MC result. Compared to Figure 5, the second-order correction on mean heads in the KLME approach is larger when the conductivity correlation lengths or variance increases. As in section 3.1.1, the head variance increases and the second-order correction becomes more significant when the conductivity correlation lengths or variance increases. In particular, when the conductivity correlation lengths increase to $10.0 \mathrm{~m}$, the second-order correction becomes so large that the second-order head variance calculated by KLME overshoots the MC result in the recharge area. For flux variance, however, the computed values do not show large variation when the conductivity correlation lengths increase from $5.0 \mathrm{~m}$ to $10.0 \mathrm{~m}$. It appears that under the conditions examined in this case, the calculated flux statistics converge and do not change further with conductivity correlation lengths after $5.0 \mathrm{~m}$. When the variance of conductivity increases (Figure 7), the secondorder correction to head variance is effective and brings about a close agreement with the MC result, and flux variance increases and the second-order correction remains insignificant in the KLME approach.

[35] It is clear from Figures 2-7 that after the addition of external stresses such as recharge, the flux statistics become dominated by the lower-order terms (i.e., zeroth and first) and the contributions of the second-order terms are much reduced. Comparing the mean flux and flux variance under the same model settings and conductivity statistics, the second-order correction is much less significant with recharge than without. As will be demonstrated in the following discussions, by increasing the number of modes at each expansion order, the first-order KLME results can be further improved modestly in certain cases, but the change on the second-order correction remains negligible. More- 
(a) Mean Head

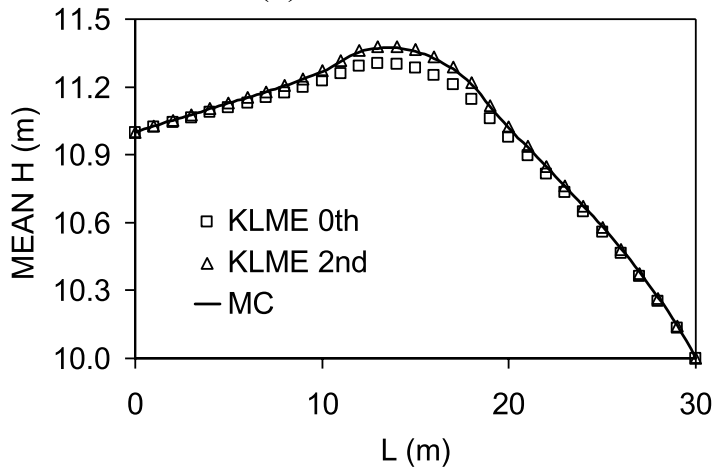

(c) Mean Flux

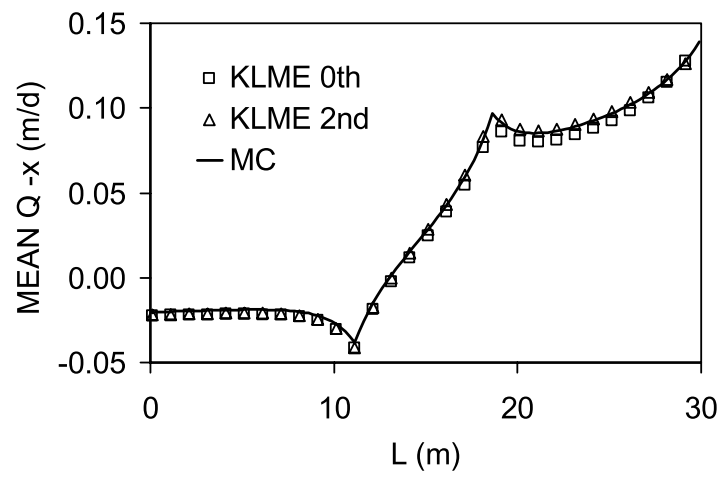

(b) Head Variance

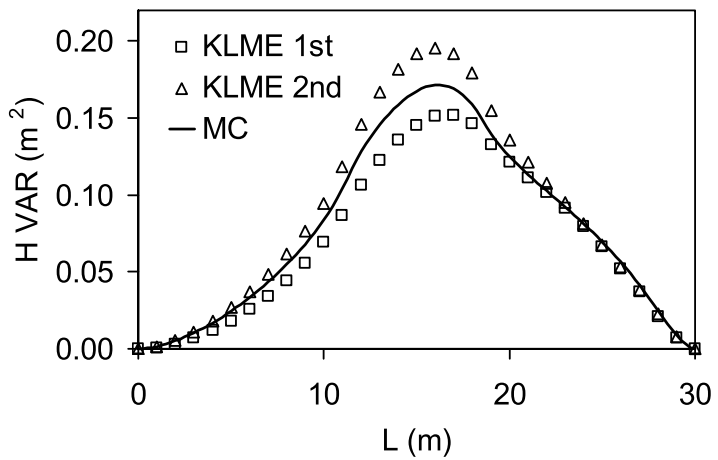

(d) Flux Variance

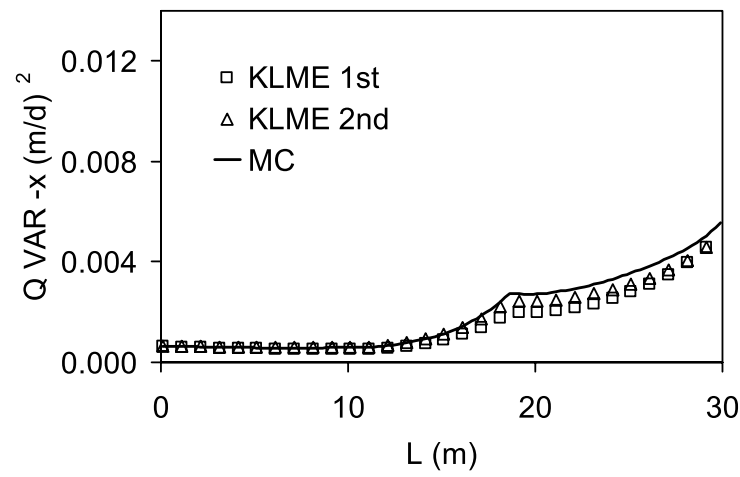

Figure 6. Mean and variance of heads and fluxes calculated along line $A-A^{\prime}$ when conductivity correlation lengths are increased to $10.0 \mathrm{~m}$ in both $x$ and $y$ directions: (a) mean head, (b) head variance, (c) mean flux, and (d) flux variance. Other model settings remain identical to those for Figure 5.

over, the first-order flux variance is more accurate in representing the $\mathrm{MC}$ results after the addition of recharge. This suggests that in the KLME approach, when deterministic external stresses predominate, one may only need to compute the zeroth-order solution to approximate mean flux and the first-order mode coefficients to approximate flux variance.

\subsubsection{Transient With Recharge}

[36] To investigate temporal effects in the flow statistics computed by the KLME approach, the test setup for Figure 5 in section 3.1.2 is conducted under transient state with a uniform specific yield of 0.1. The KLME zeroth-order head solutions from the case in section 3.1.1 are used as the initial heads in both KLME and MC transient simulations. The total simulation time is 100 day as preliminary model runs indicated that the head solutions do not show much change beyond this time frame. Simulation is divided into five time steps that follow a geometric progression with a successive size multiplier of 2.0 .

[37] Figure 8 shows the mean and variance of heads and $x$ fluxes computed by KLME and MC along $\mathrm{A}-\mathrm{A}^{\prime}$ at three different time levels $t_{1}, t_{2}$ and $t_{5}$, where $t_{1}=3.2$ day, $t_{2}=$ 9.7 day, and $t_{5}=100$ day. For illustration, only the secondorder KLME results are presented. Because of the continuous recharge inflow, the mean and variance of heads and fluxes increase with time. It is noteworthy that the two local peaks in the mean flux profiles are generated by recharge right after the start of model simulation and their values remain essentially unchanged during the temporal evolution of flow field. For all flow statistics, the KLME approach is able to provide effective representations of MC results at different times.

\subsubsection{Mode Numbers in the KLME Approach}

[38] At the beginning of section 3.1 we have mentioned that in all cases presented above, without considering the specific model settings and statistics of underlying random conductivity field, the mode numbers at different expansion orders are specified a priori and remain unchanged. The number of coefficients $h_{i_{1}, i_{2}, \ldots, i_{m}}^{(m)}$ calculated in the KLME approach is about one tenth of the total number of realizations in $\mathrm{MC}$ simulations. Additional computational efficiency of KLME approach in certain applications can be demonstrated by further examining the results with different number of modes. Here we take Figure 2 as an example, where the first-order results provide an accurate approximation on both head and flux variances and the secondorder correction is found to be small and may be practically negligible (which is generally the case when the correlation lengths relative to physical domain size and the variance of underlying conductivity field are sufficiently small). Figure 9 plots the first-order variance of heads and fluxes at different modes for the model studied in Figure 2 where $\eta_{x}=\eta_{y}=5.0 \mathrm{~m}$ and $\sigma_{Y}^{2}=1$. The firstorder head variance converges quickly after using only the first 5 modes. For flux variance, the result is more sensitive to the number of modes and requires about the first 50 
(a) Mean Head

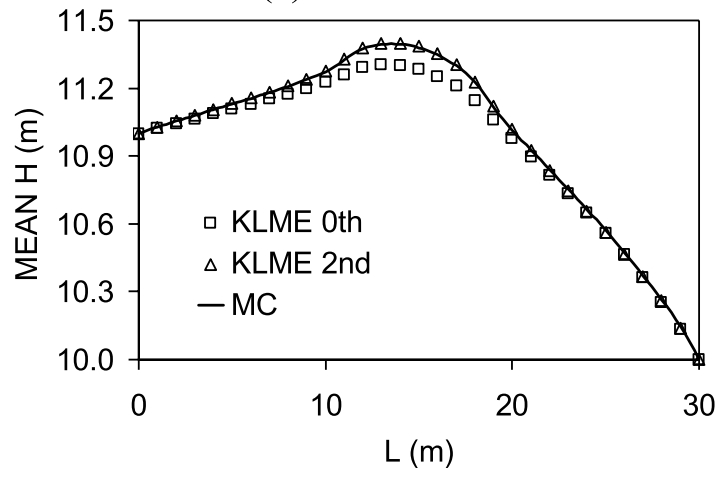

(c) Mean Flux

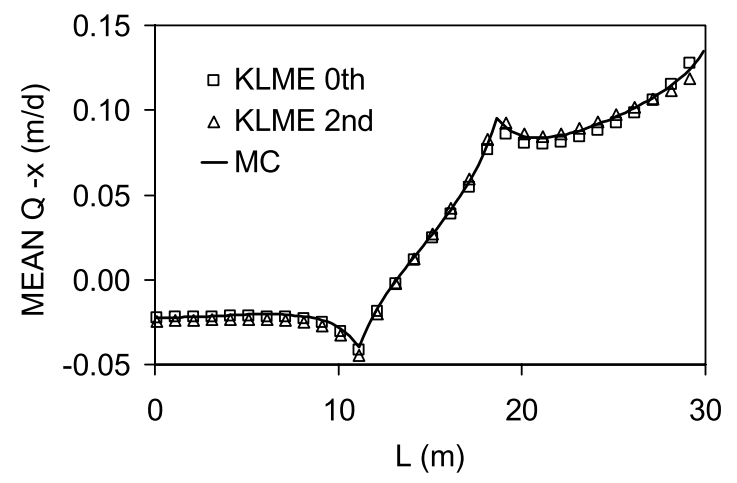

(b) Head Variance

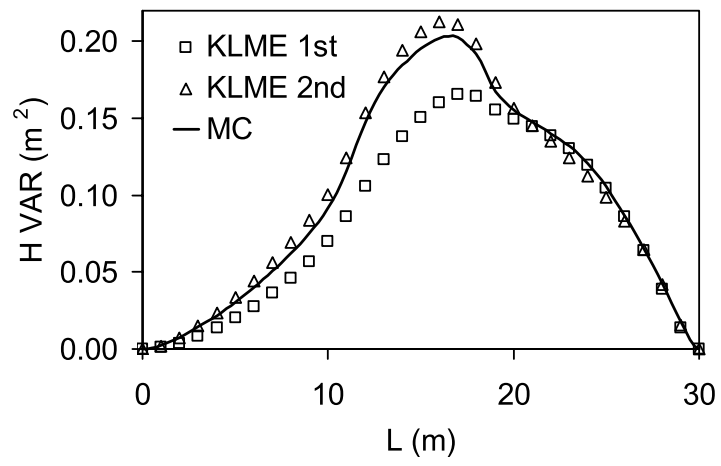

(d) Flux Variance

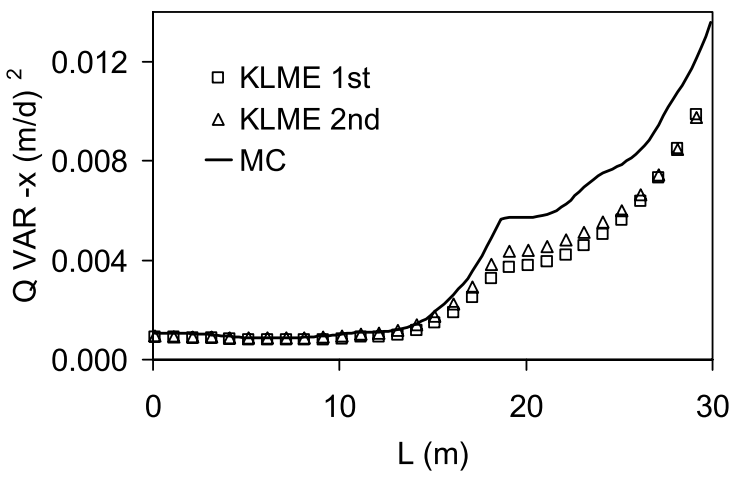

Figure 7. Mean and variance of heads and fluxes calculated along line A-A' when conductivity variance is increased to 2.0: (a) mean head, (b) head variance, (c) mean flux, and (d) flux variance. Other model settings remain identical to those for Figure 5.

modes to obtain an adequate approximation. Clearly, computational effort can be further reduced in the KLME approach by accommodating only those modes that are contributing significantly.

[39] Further investigations show that although the head statistics generally converge rather well for the number of modes selected (i.e., 100 modes at the first order, $20 \times 20$ at the second and $10 \times 10 \times 10$ at the third), an even higher number of modes may further improve the accuracy of flux statistics under certain circumstances. Figures $10 \mathrm{a}$ and $10 \mathrm{~b}$ provide the head and flux variance calculated using two different sets of mode numbers in the KLME approach for the same model setting as in Figure 4 where $\sigma_{Y}^{2}=2$. The flux variance calculated by MC with 5000 realizations was not stable. After the number of realizations is increased to 10000 , the accuracy of MC results is improved. In the KLME approach, there is no obvious difference on head variance after the mode numbers are increased from (100, $20 \times 20,10 \times 10 \times 10)$, indicated as M1, to M2 $(200,40 \times$ $40,30 \times 30 \times 30$ ), at the first, second and third orders, respectively. This is because the head statistics have already converged for the first three orders with the smaller mode numbers. For flux variance, however, the first-order KLME results show some improvement after the increase of mode number at the first order, while the second-order correction shows little change. Note that the improvement on the second-order KLME results at the larger mode numbers is mainly due to the change in the first-order values rather than that in the second-order correction. In this particular example, a combination of $(200,20 \times 20,10 \times 10 \times 10)$ modes at the first three orders appears sufficient to approximate the flux variance as further increasing the mode numbers shows little change on the results. To further enhance solute accuracy, even higher-order correction terms may be needed. On the basis of the early work of $L u$ and Zhang [2004a], for a moderate variability in log conductivity the contributions of high-order terms will decrease as the expansion order goes up; for extremely large variabilities, the high-order terms may bring about larger contributions than do the lower-order ones.

[40] As mentioned earlier, the contributions of higherorder terms become small when the correlation lengths decrease. Figures $10 \mathrm{c}$ and $10 \mathrm{~d}$ demonstrate that this is so even when the variance of log conductivity is large $\left(\sigma_{Y}^{2}=2\right.$, which is equivalent to the coefficient of variation for hydraulic conductivity equal to $253 \%$ ). After the correlation lengths are decreased from $5.0 \mathrm{~m}$ to $1.0 \mathrm{~m}$, the observation that the second-order results are essentially the same as the first-order ones at each mode combination indicates that the first-order values are more dominant and the second-order correction becomes negligible, especially in the flux variance case. On the other hand, a larger number of modes (i.e., 600) are needed at the first expansion order to better characterize flux statistics as revealed by sensitivity runs. Overall, the accuracy of KLME approach improves for both head and flux variances as the correlation lengths decrease, 
(a) Mean Head

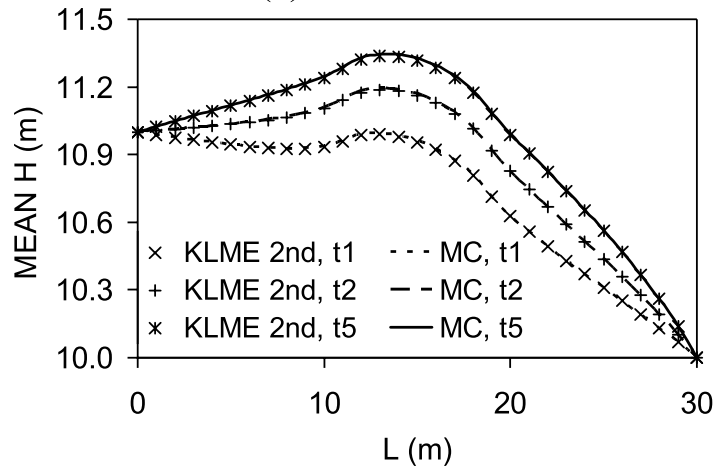

(c) Mean Flux

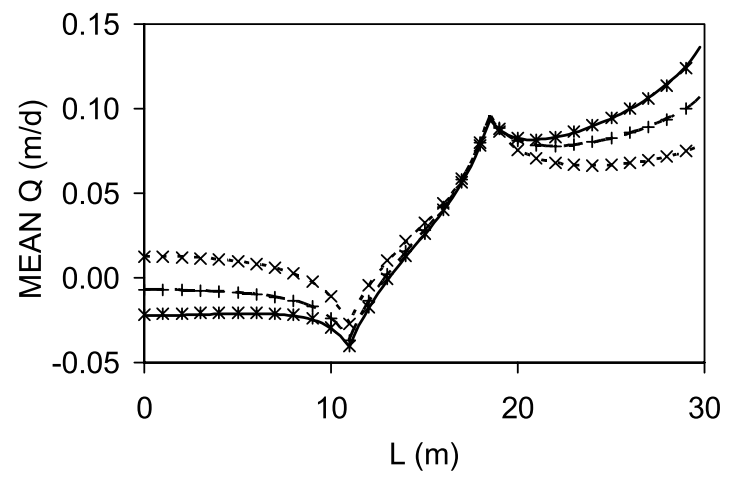

(b) Head Variance

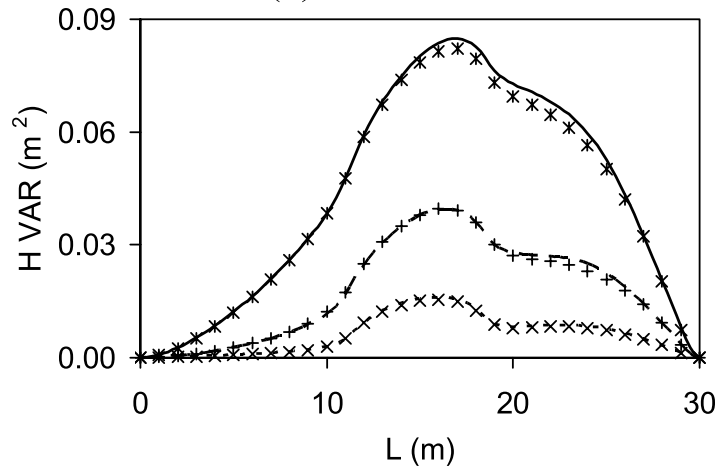

(d) Flux Variance

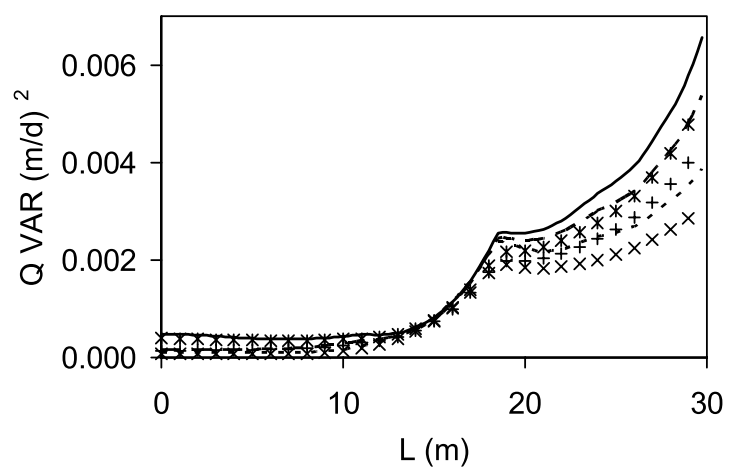

Figure 8. Mean and variance of heads and fluxes calculated at three different times along line $\mathrm{A}-\mathrm{A}^{\prime}$ in the 2-D transient example with recharge: (a) mean head, (b) head variance, (c) mean flux, and (d) flux variance. The legend for different times in Figures $8 \mathrm{~b}, 8 \mathrm{c}$, and $8 \mathrm{~d}$ is shown in Figure 8a.

and the computational effort is about the same since the second- and higher-order corrections may not be needed although more modes are needed in computing the firstorder term.

\subsection{Three-Dimensional Unconfined Flow}

[41] In this example we evaluated the KLME approach in a 3-D unconfined flow system. The schematic diagram of model setup is shown on Figure 11. A 3-D $30 \mathrm{~m}$ long by $10 \mathrm{~m}$ wide by $9 \mathrm{~m}$ deep rectangular parallelepiped is discretized into a block-centered finite difference mesh of 121 columns by 40 rows by 3 layers. Each cell is $0.25 \mathrm{~m}$ long, $0.25 \mathrm{~m}$ wide and $3 \mathrm{~m}$ deep. The western and eastern boundaries have constant heads of $8.5 \mathrm{~m}$ and $7.5 \mathrm{~m}$, respectively. The origin of coordinate system is at the upper, left and top corner of the domain, with $z$ direction pointing downward. There is no flow across the north, south and bottom borders. A local recharge is applied at rate $0.3 \mathrm{~m} / \mathrm{d}$ in a region of 10 rows by 30 columns ( $x$ : $11.125-18.625 \mathrm{~m} ; y: 3.75-$ $6.25 \mathrm{~m}$ ). Here the recharge rate is assigned a high value such that its effects can be demonstrated better. There is a well located at the center of domain (column 61, row 21, and layer 2) and pumps at a volumetric rate of $3 \mathrm{~m}^{3} / \mathrm{d}$. Three total stress periods are used. The first stress period is steady state without recharge or pumping well. In the second stress period recharge is included and simulation is conducted in a transient state. In the third stress period simulation is transient again and both recharge and pumping well are considered. The length of first stress period is 1 day. The second period is 10 days and divided into five time steps with a geometric multiplier of 2.0. The third period is 5 days and divided into five time steps with a multiplier of 3.0. The log hydraulic conductivity field has an unconditional zero mean $\left(K_{G}=1.0 \mathrm{~m} /\right.$ day $)$, variance $\sigma_{Y}^{2}=1.0$ and correlation lengths $\eta_{x}=\eta_{y}=\eta_{z}=5.0 \mathrm{~m}$. Again, in the KLME approach we obtained the solutions of $h_{i_{1}, i_{2}, \ldots, i_{m}}^{(m)}$ up to the third order, 100 modes at the first order, 20 by 20 modes at the second order, and 10 by 10 by 10 modes at the third order. In the MC simulations 5000 realizations are used.

[42] Figure 12 displays the mean and variance of heads and fluxes at the end of the third stress period. Results are shown along the 20th row of top unconfined layer. Compared to the 2-D cases, the mean of heads is not as curving and head variance is not as skewed toward downstream. This is because of the vertical flow communication between the top unconfined layer and underlying confined portions in the 3-D model. It is seen that recharge (positive inflow) has raised the values of all flow statistics, while well pumping (negative outflow) reduces the calculated values and creates a local dent on both head and flux variance curves. For the mean of heads and fluxes, the KLME and $\mathrm{MC}$ results are in close agreement. For head variance, the first-order values calculated by KLME are lower than the $\mathrm{MC}$ results in the vicinity of recharge and the second-order correction is able to improve significantly solution accuracy. For flux variance, there is a noticeable mismatch between the $\mathrm{MC}$ and the first-order KLME results, and the second- 
(a) Head Variance

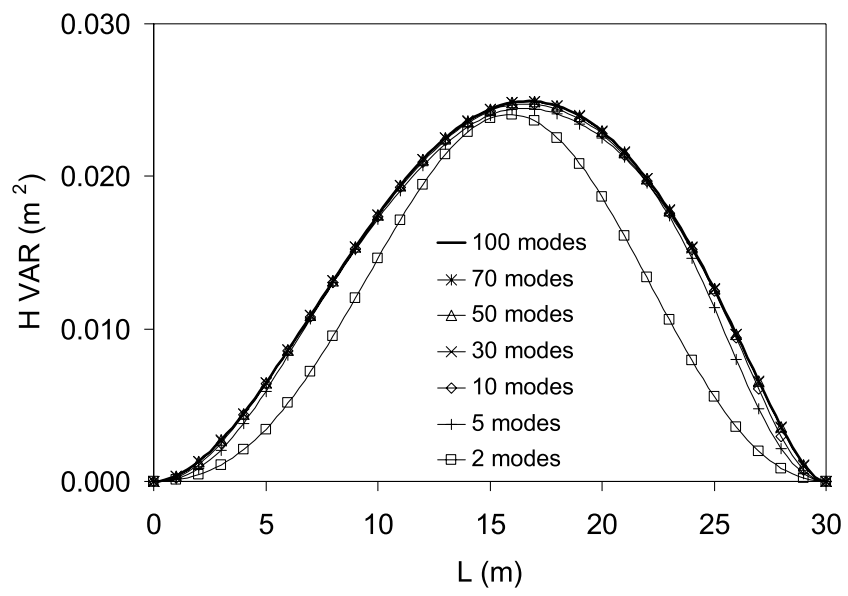

(b) Flux Variance

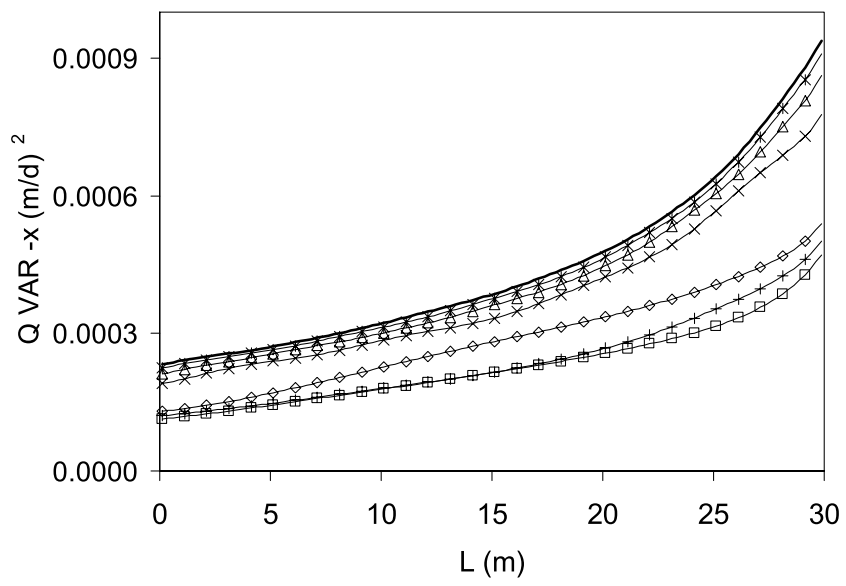

Figure 9. The first-order (a) head variance and (b) flux variance calculated by the KLME approach with different number of modes. Model settings are identical to those for Figure 2. The legend for different modes in Figure $9 \mathrm{~b}$ is shown in Figure 9a.

order KLME correction is insignificant. Nonetheless, the general trend of flux variance is the same from the KLME and the MC approaches. It is noteworthy that the mismatch of head variance occurs mainly in recharge area; by contrast, for the flux variance, the mismatch appears noticeably right after recharge and continues to remain large throughout the entire downstream area.

\section{Summary and Conclusions}

[43] This paper addresses the issue of parameter uncertainty associated with hydraulic conductivity field in modeling of unconfined flow systems. Here we adopt the Karhunen-Loeve decomposition-based moment equation (KLME) approach of Zhang and Lu [2004], and extend it to nonlinear, unconfined flow situations. In this approach, the log-transformed hydraulic conductivity field is first expanded into a series in terms of orthogonal Gaussian standard random variables. Next, head is decomposed as a perturbation expansion series in which each individual term is further expanded into a polynomial series of orthogonal Gaussian standard random variable products. The coefficients associated with head expansions are solved recursively. The expanded equations are reformulated such that they share the same structure as the original deterministic governing equation and therefore any existing flow simulator such as the USGS groundwater model MODFLOW-2000 can be used. Finally, the statistics of head and flux are computed using some simple algebraic operations on these coefficients. A new code called "MODFLOW-STO" has been developed to implement numerically the stochastic formulation presented in this work.

[44] The KLME approach has been evaluated against traditional $\mathrm{MC}$ simulation technique in two hypothetical numerical experiments. The first test considers a 2-D unconfined flow system in which simulations are conducted under different conditions: (1) steady state, no recharge, (2) steady state with recharge, and (3) transient with recharge. The second test is on a 3-D unconfined flow system with three different stress periods and results are provided for the third stress period in which simulation is transient and both recharge and a pumping well are included. In all cases, the KLME approach is able to provide an effective solution to the mean of heads and fluxes, the head variance, and to a less extent, the flux variance. Transient simulation results indicate that the KLME approach is able to capture the evolution of flow statistics with time.

[45] Some specific conclusions can be drawn from the two examples in this work. First, when the correlation lengths relative to physical domain size and variance of the random conductivity field increase, the variance of heads and fluxes generally increases and higher-order correction becomes more significant in the KLME approach. In situations like the case in Figure 7 where recharge has a controlling effect on flow field, flux variance does not show much change after correlation lengths are beyond certain level. Second, the head statistics can be accurately approximated using a very small number of modes in the KLME approach. The flux results, however, appear more sensitive and require more modes to converge particularly at the first order. When the conductivity variance and correlation lengths are large, higher-order terms may be needed to further enhance solution accuracy. Third, external sink/source terms such as recharge and pumping well can raise the variance of heads and fluxes. In systems containing multiple sinks/sources, the calculated flow statistics are dependent on their net results. Fourth, when external sinks/sources have a controlling effect on flow field, they also dominate the mismatch between KLME and MC. For the calculated head variance, the major mismatch always appears near the local recharge area (pumping well located within recharge area). For flux variance, the mismatch becomes noticeable right after recharge and persists throughout the downstream region. Fifth, in the external sink/source area where the difference between the MC and first-order KLME approaches is large, the second-order KLME correction becomes more significant on both the mean and variance of heads. However, the second-order correction on flux statistics is less significant as the zeroth- and first-order results become more accurate and dominant. This suggests that in the KLME approach, when deterministic external stresses predominate, one may 
(a) Head Variance, $\eta_{x}=\eta_{y}=5.0 \mathrm{~m}$

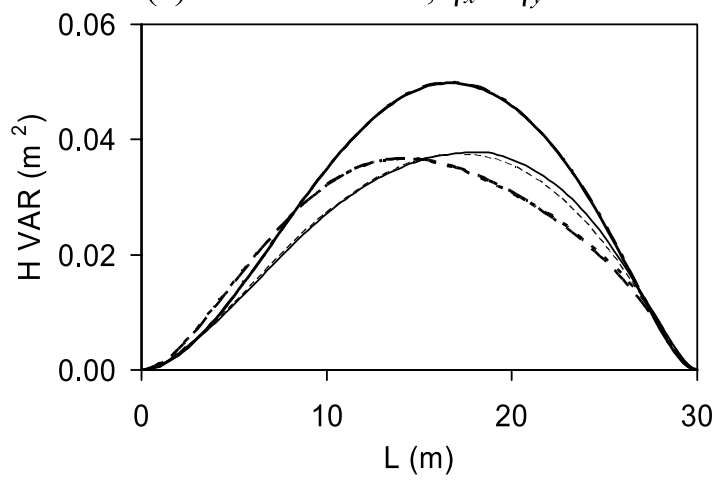

(c) Head Variance, $\eta_{x}=\eta_{y}=1.0 \mathrm{~m}$

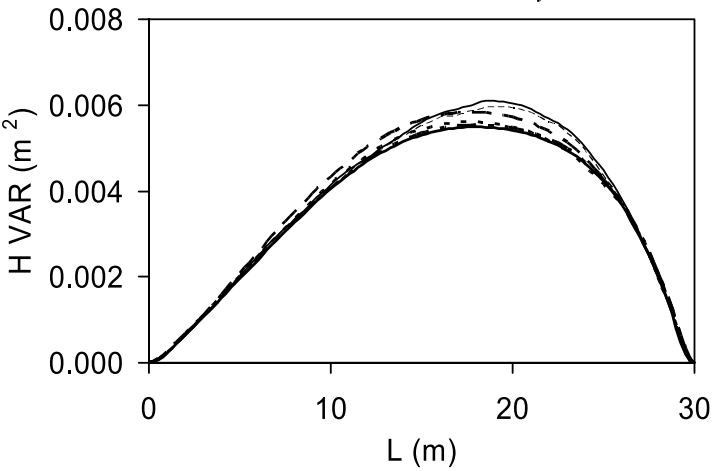

(b) Flux Variance, $\eta_{x}=\eta_{y}=5.0 \mathrm{~m}$

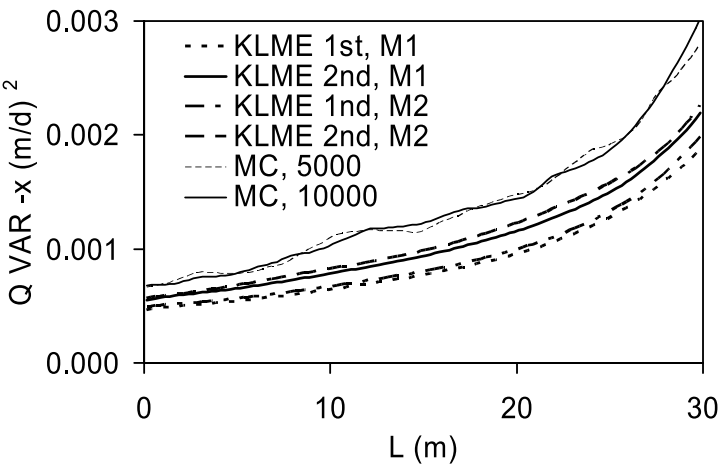

(d) Flux Variance, $\eta_{x}=\eta_{y}=1.0 \mathrm{~m}$

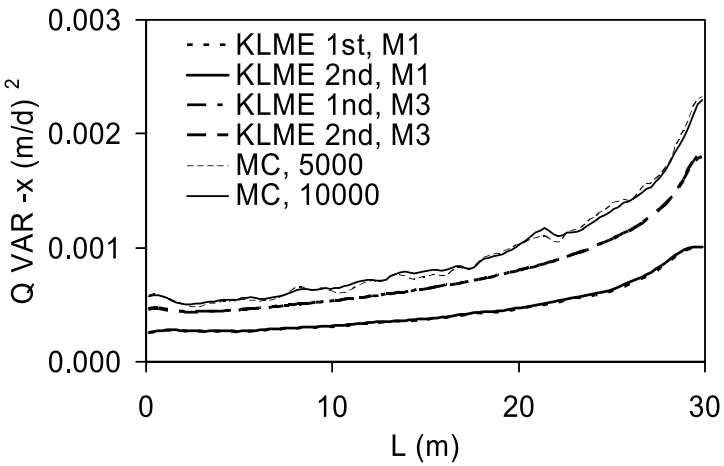

Figure 10. Variance of heads and fluxes calculated by MC and the KLME approach with different mode numbers and correlation lengths: (a) head variance, $\eta_{x}=\eta_{y}=5.0 \mathrm{~m}$, (b) flux variance, $\eta_{x}=\eta_{y}=$ $5.0 \mathrm{~m},(\mathrm{c})$ head variance, $\eta_{x}=\eta_{y}=1.0 \mathrm{~m}$, and (d) flux variance, $\eta_{x}=\eta_{y}=1.0 \mathrm{~m}$. The legend for different curves in Figures 10a and 10c is shown in Figures 10b and 10d, respectively. M1 stands for the mode numbers $(100,20 \times 20,10 \times 10 \times 10)$, M2 stands for $(200,40 \times 40,30 \times 30 \times 30)$, and M3 stand for $(600,40 \times 40,30 \times 30 \times 30)$ at the first three expansion orders. Model settings are identical to those for Figure 4. In the MC simulations, 5000 and 10,000 realizations are used. The log conductivity variance is 2.0 .

only need to compute the zeroth-order solution to approximate mean flux and the first-order mode coefficients to approximate the flux variance. Sixth, compared to 2-D case, the nonlinear features of flow statistics in a 3-D system is less pronounced due to the vertical flow communication between top unconfined layer and underlying confined portions.

[46] A final note worth emphasizing is the computational efficiency of KLME approach as compared to the traditional MC technique. In all different test cases presented in section 3, the KLME approach required much less CPU run time than did MC simulations. This is because the total number of mode coefficients is much less than the total number of realizations in $\mathrm{MC}$ simulations. When the correlation length is small relative to the domain size, second- and higher-order corrections are not needed although it requires a relatively large number of modes to accurately compute the first-order solutions; when the correlation length is large, the number of modes required at each expansion order is small although second- or higher-order corrections may be needed. In the case of moderate variance of log hydraulic conductivity, the firstor second-order terms provide accurate solutions; in the case of large variance, higher-order corrections may be needed, resulting in a larger computational effort. On the other hand, in the MC approach the computational effort also increases with the magnitude of the variance in order to achieve statistical convergence. Moreover, as the structure of expanded equations is the same at different orders and modes, the left-hand side coefficient matrix involved

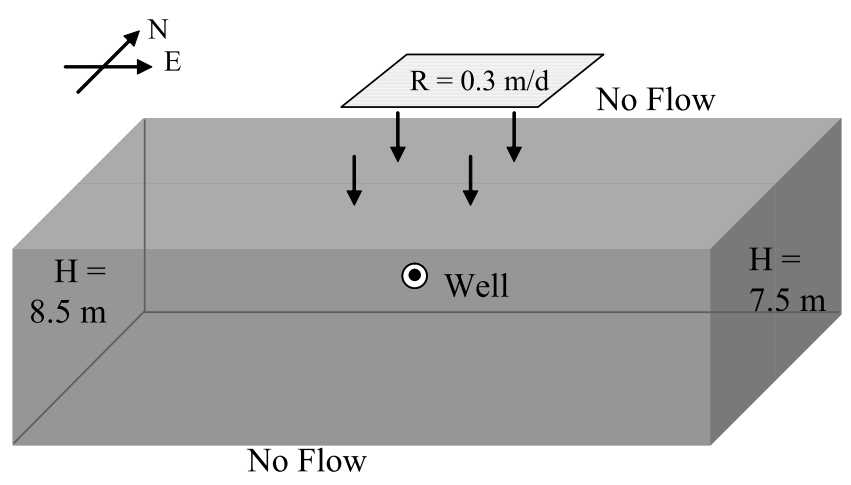

Figure 11. Schematic diagram of model setup in the 3-D unconfined flow problem. 
(a) Mean Head

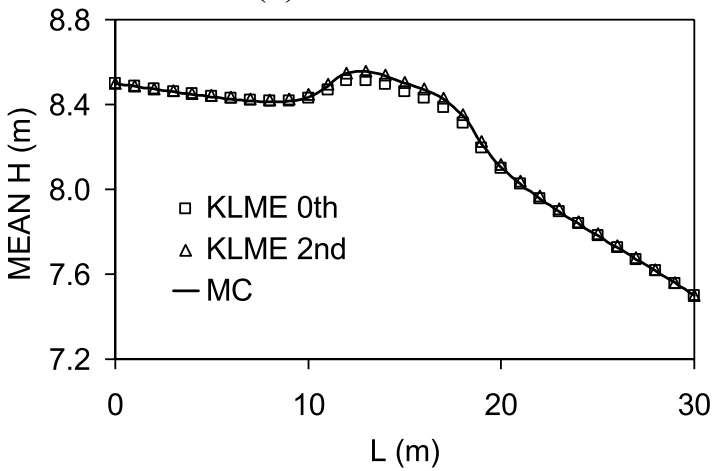

(c) Mean Flux

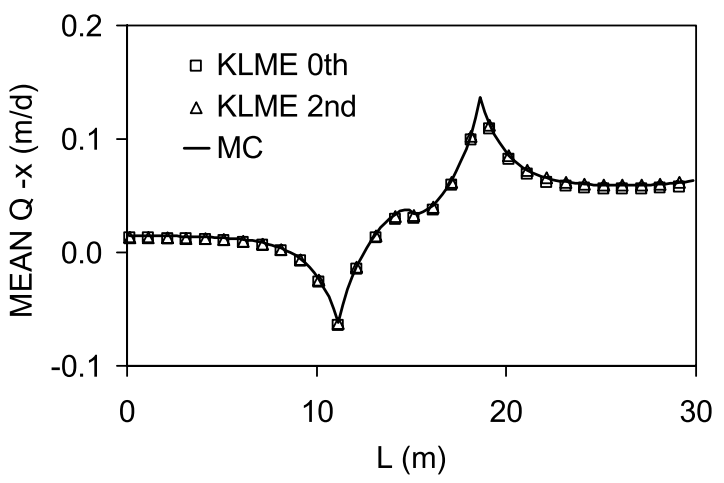

(b) Head Variance

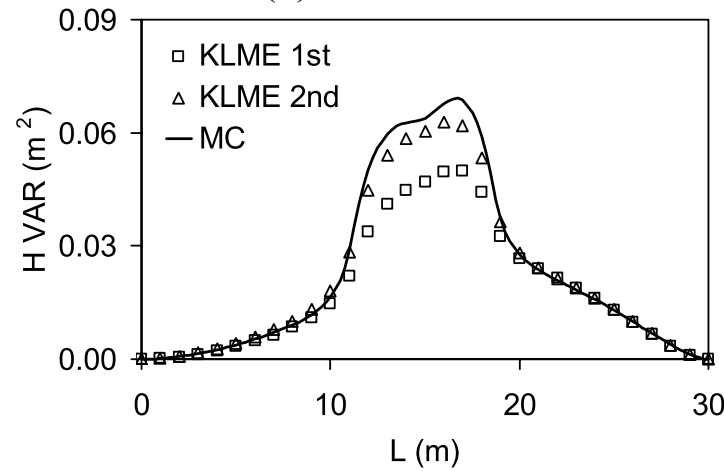

(d) Flux Variance

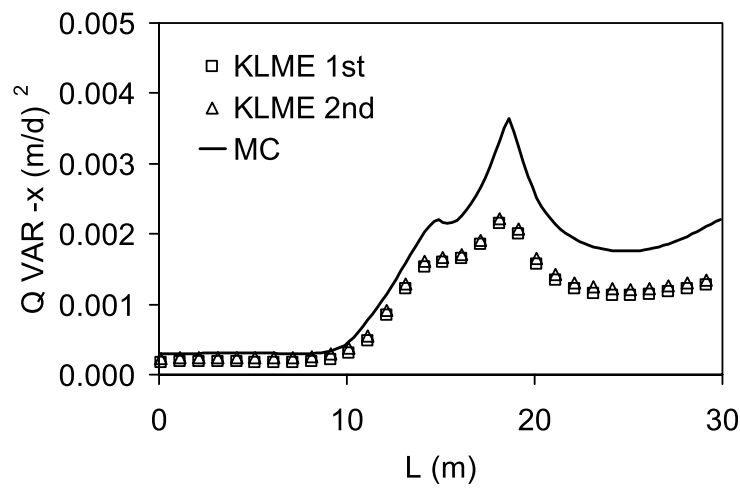

Figure 12. Mean and variance of heads and fluxes calculated along row 20 of top unconfined layer at the end of third stress period in the 3-D example: (a) mean head, (b) head variance, (c) mean flux, and (d) flux variance. The third stress period is transient with both recharge and a pumping well.

in each MODFLOW-2000 subroutine calculation remains identical. This feature can further allow us to significantly reduce computational effort required by the KLME approach. Because of its accuracy and computational advantages, the KLME approach is expected to provide a tool for conducting uncertainty analysis in subsurface flow modeling under general field conditions in an efficient, effective manner.

\section{Appendix A}

[47] Substituting expansions of (3), (7) and $h_{B}(\boldsymbol{x}, t)$ into (2) and separating terms at different orders, one obtains a set of equations for different orders of head terms. The firstorder equations are shown in (9). The second-order equations for the top water table layer and the underlying confined portions are given as

$$
\begin{gathered}
\nabla_{x y} \cdot\left[K_{G} h_{B}^{(0)} \nabla_{x y} h^{(2)}\right]+K_{G} \frac{\partial h^{(2)}}{\partial z}+g_{u}^{(2)}=S_{y} \frac{\partial h^{(2)}}{\partial t}, \\
\nabla \cdot\left[K_{G} \nabla h^{(2)}\right]+g_{c}^{(2)}=S_{S} \frac{\partial h^{(2)}}{\partial t}
\end{gathered}
$$

subject to initial and boundary conditions

$$
\begin{aligned}
& h^{(2)}(\boldsymbol{x}, 0)=0, \quad x \in D, \\
& h^{(2)}(\boldsymbol{x}, t)=0, \quad \boldsymbol{x} \in \Gamma_{D},
\end{aligned}
$$

$$
K_{G} \nabla h^{(2)}(\boldsymbol{x}, t) \cdot \boldsymbol{n}(\boldsymbol{x})=-\frac{1}{2} Y^{\prime 2} Q(\boldsymbol{x}, t), \quad \boldsymbol{x} \in \Gamma_{N},
$$

where

$$
\begin{gathered}
g_{u}^{(2)}=\nabla_{x y} \cdot\left[K_{G}\left(\frac{Y^{\prime 2}}{2} h_{B}^{(0)}+Y^{\prime} h^{(1)}+h^{(2)}\right) \nabla_{x y} h^{(0)}+K_{G}\right. \\
\left.\cdot\left(Y^{\prime} h_{B}^{(0)}+h^{(1)}\right) \nabla_{x y} h^{(1)}\right]+K_{G}\left(Y^{\prime} \frac{\partial h^{(1)}}{\partial z}+\frac{Y^{\prime 2}}{2} \frac{\partial h^{(0)}}{\partial z}\right) \\
g_{c}^{(2)}=\nabla \cdot\left[K_{G}\left(Y^{\prime} \nabla h^{(1)}+\frac{Y^{\prime 2}}{2} \nabla h^{(0)}\right)\right]
\end{gathered}
$$


Similarly, the third-order equations the top water table layer and the underlying confined portions are respectively given as

$$
\begin{gathered}
\nabla_{x y} \cdot\left[K_{G} h_{B}^{(0)} \nabla_{x y} h^{(3)}\right]+K_{G} \frac{\partial h^{(3)}}{\partial z}+g_{u}^{(3)}=S_{y} \frac{\partial h^{(3)}}{\partial t}, \\
\nabla \cdot\left[K_{G} \nabla h^{(3)}\right]+g_{c}^{(3)}=S_{S} \frac{\partial h^{(3)}}{\partial t},
\end{gathered}
$$

subject to initial and boundary conditions,

$$
\begin{gathered}
h^{(3)}(\boldsymbol{x}, 0)=0, \quad x \in D, \\
h^{(3)}(\boldsymbol{x}, t)=0, \quad \boldsymbol{x} \in \Gamma_{D}, \\
K_{G} \nabla h^{(3)}(\boldsymbol{x}, t) \cdot \boldsymbol{n}(\boldsymbol{x})=\frac{1}{6} Y^{\prime 3} Q(\boldsymbol{x}, t), \quad \boldsymbol{x} \in \Gamma_{N},
\end{gathered}
$$

where

$$
\begin{aligned}
g_{u}^{(3)}= & \nabla_{x y} \cdot\left[K_{G}\left(\frac{Y^{\prime 3}}{6} h_{B}^{(0)}+\frac{Y^{\prime 2}}{2} h^{(1)}+Y^{\prime} h^{(2)}\right) \nabla_{x y} h^{(0)}+K_{G}\right. \\
& \left.\cdot\left(\frac{Y^{\prime 2}}{2} h_{B}^{(0)}+Y^{\prime} h^{(1)}\right) \nabla_{x y} h^{(1)}+K_{G}\left(Y^{\prime} h_{B}^{(0)}+h^{(1)}\right) \nabla_{x y} h^{(2)}\right] \\
& +K_{G}\left(Y^{\prime} \frac{\partial h^{(2)}}{\partial z}+\frac{Y^{\prime 2}}{2} \frac{\partial h^{(1)}}{\partial z}+\frac{Y^{\prime 3}}{6} \frac{\partial h^{(0)}}{\partial z}\right), \\
g_{c}^{(3)} & =\nabla \cdot\left[K_{G}\left(Y^{\prime} \nabla h^{(2)}+\frac{Y^{\prime 2}}{2} \nabla h^{(1)}+\frac{Y^{\prime 3}}{6} \nabla h^{(0)}\right)\right] . \quad(\mathrm{A} 14)
\end{aligned}
$$

In general, at $m$ th order, $m \geq 1$,

$$
\begin{gathered}
\nabla_{x y} \cdot\left[K_{G} h_{B}^{(0)} \nabla_{x y} h^{(m)}\right]+K_{G} \frac{\partial h^{(m)}}{\partial z}+g_{u}^{(m)}=S_{y} \frac{\partial h^{(m)}}{\partial t}, \\
\nabla \cdot\left[K_{G} \nabla h^{(m)}\right]+g_{c}^{(m)}=S_{S} \frac{\partial h^{(m)}}{\partial t}
\end{gathered}
$$

subject to initial and boundary conditions,

$$
\begin{gathered}
h^{(m)}(\boldsymbol{x}, 0)=0, \quad \boldsymbol{x} \in D, \\
h^{(m)}(\boldsymbol{x}, t)=0, \quad \boldsymbol{x} \in \Gamma_{D}, \\
K_{G} \nabla h^{(m)}(\boldsymbol{x}, t) \cdot \boldsymbol{n}(\boldsymbol{x})=\frac{(-1)^{m+1}}{m !} Y^{\prime m} Q(\boldsymbol{x}, t), \boldsymbol{x} \in \Gamma_{N},
\end{gathered}
$$

where

$$
\begin{aligned}
g_{u}^{(m)}= & \nabla_{x y} \cdot\left[K_{G} \sum_{k=1}^{m} \sum_{i=0}^{k} \frac{Y^{\prime k-i}}{(k-i) !} h_{B}^{(i)} \nabla_{x y} h^{(m-k)}\right] \\
& +K_{G} \sum_{k=1}^{m} \frac{Y^{\prime k}}{k !} \frac{\partial h^{(m-k)}}{\partial z},
\end{aligned}
$$

$$
g_{c}^{(m)}=\nabla \cdot\left[\sum_{k=1}^{m} K_{G} \frac{Y^{\prime k}}{k !} \nabla h^{(m-k)}\right] .
$$

\section{Appendix B}

[48] Substituting (10) and (6b) into (9) and recalling the fact that the set $\left\{\xi_{i}\right\}$ are orthogonal, one obtains equations for the mode coefficients $h_{i_{1}, i_{2}, \ldots, i_{m}}^{(m)}$ at different orders $m$. The equations for first-order mode coefficients $h_{i}^{(1)}$ are shown in (11). The equations for the second-order mode coefficients $h_{i, j}^{(2)}$ are given as,

$$
\nabla_{x y} \cdot\left[K_{G} h_{B}^{(0)} \nabla_{x y} h_{i, j}^{(2)}\right]+K_{G} \frac{\partial h_{i, j}^{(2)}}{\partial z}+g_{u_{i, j}}^{(2)}=S_{y} \frac{\partial h_{i, j}^{(2)}}{\partial t},
$$

$$
\nabla \cdot\left[K_{G} \nabla h_{i, j}^{(2)}\right]+g_{c_{i, j}}^{(2)}=S_{S} \frac{\partial h_{i, j}^{(2)}}{\partial t}
$$

subject to initial and boundary conditions,

$$
\begin{gathered}
h_{i, j}^{(2)}(\boldsymbol{x}, 0)=0, \quad \boldsymbol{x} \in D, \\
h_{i, j}^{(2)}(\boldsymbol{x}, t)=0, \quad \boldsymbol{x} \in \Gamma_{D}, \\
K_{G} \nabla h_{i, j}^{(2)}(\boldsymbol{x}, t) \cdot \boldsymbol{n}(\boldsymbol{x})=-\frac{1}{2} f_{i} f_{j} Q(\boldsymbol{x}, t), \quad \boldsymbol{x} \in \Gamma_{N},
\end{gathered}
$$

where

$$
\begin{aligned}
g_{u_{i, j}}^{(2)}= & \nabla_{x y} \cdot\left[K_{G}\left(\frac{f_{i} f_{j}}{2} h_{B}^{(0)}+\frac{f_{i} h_{j}^{(1)}+f_{j} h_{i}^{(1)}}{2}+h_{i, j}^{(2)}\right) \cdot \nabla_{x y} h^{(0)}\right. \\
& \left.+K_{G}\left(\frac{f_{i} h_{B}^{(0)}+h_{i}^{(1)}}{2} \nabla_{x y} h_{j}^{(1)}+\frac{f_{j} h_{B}^{(0)}+h_{j}^{(1)}}{2} \nabla_{x y} h_{i}^{(1)}\right)\right] \\
& +K_{G}\left(f_{i} \frac{\partial h_{j}^{(1)}}{\partial z}+f_{j} \frac{\partial h_{i}^{(1)}}{\partial z}\right) / 2+K_{G} \frac{f_{i} f_{j}}{2} \frac{\partial h^{(0)}}{\partial z}, \\
& g_{c_{i, j}^{(2)}}=\nabla \cdot\left[K_{G}\left(f_{i} \nabla h_{j}^{(1)}+f_{j} \nabla h_{i}^{(1)}+f_{i} f_{j} \nabla h^{(0)}\right) / 2\right] .
\end{aligned}
$$

Note that in (B6) and (B7), to maintain the symmetry of $h_{i, j}^{(2)}$ with respect to its indices, we have written the coefficient of $Y^{\prime} h^{(1)}$ at mode $i, j$ in the form of $\left(f_{i} h_{j}^{(1)}+f_{j} h_{i}^{(1)}\right) / 2$. Similar treatment has been applied to the coefficients of $Y^{\prime} \nabla_{x y} h^{(1)}$, $h^{(1)} \nabla_{x y} h^{(1)}, Y^{\prime} \partial h^{(1)} / \partial z$, and $Y^{\prime} \nabla h^{(1)}$. Moreover, due to the symmetry, one only needs to solve $h_{i, j}^{(2)}$ for $i \leq j$ as $h_{i, j}^{(2)}$ for $i>j$ can be directly obtained by simple manipulation of the subscripts. The equations for the third-order mode coefficients $h_{i, j, k}^{(3)}$ can be similarly written as,

$$
\nabla_{x y} \cdot\left[K_{G} h_{B}^{(0)} \nabla_{x y} h_{i, j, k}^{(3)}\right]+K_{G} \frac{\partial h_{i, j, k}^{(3)}}{\partial z}+g_{u_{i, j, k}}^{(3)}=S_{y} \frac{\partial h_{i, j, k}^{(3)}}{\partial t}
$$




$$
\nabla \cdot\left[K_{G} \nabla h_{i, j, k}^{(3)}\right]+g_{c_{i, j, k}}^{(3)}=S_{S} \frac{\partial h_{i, j, k}^{(3)}}{\partial t},
$$

subject to initial and boundary conditions,

$$
\begin{gathered}
h_{i, j, k}^{(3)}(\boldsymbol{x}, 0)=0, \quad \boldsymbol{x} \in D, \\
h_{i, j, k}^{(3)}(\boldsymbol{x}, t)=0, \quad \boldsymbol{x} \in \Gamma_{D}, \\
K_{G} \nabla h_{i, j, k}^{(3)}(\boldsymbol{x}, t) \cdot \boldsymbol{n}(\boldsymbol{x})=-\frac{1}{6} f_{i} f_{j} f_{k} Q(\boldsymbol{x}, t), \\
\boldsymbol{x} \in \Gamma_{N},
\end{gathered}
$$

where

$$
\begin{aligned}
g_{u_{i, j, k}}^{(3)}= & \nabla_{x y} \cdot\left[K _ { G } \left(\frac{f_{i} f_{j} f_{k}}{6} h_{B}^{(0)}+\frac{f_{i} f_{j} h_{k}^{(1)}+f_{i} f_{k} h_{j}^{(1)}+f_{j} f_{k} h_{i}^{(1)}}{6}\right.\right. \\
& +\frac{\left.\left.f_{i} h_{j, k}^{(2)}+f_{j} h_{i, k}^{(2)}+f_{k} h_{i, j}^{(2)}+h_{i, j, k}^{(3)}\right)\right] \nabla_{x y} h^{(0)}}{3} \\
& +K_{G}\left(\frac{f_{i} f_{j}}{6} h_{B}^{(0)}+\frac{f_{i} h_{j}^{(1)}+f_{j} h_{i}^{(1)}}{6}+\frac{h_{i, j}^{(2)}}{3}\right) \cdot \nabla_{x y} h_{k}^{(1)} \\
& +K_{G}\left(\frac{f_{i} f_{k}}{6} h_{B}^{(0)}+\frac{f_{i} h_{k}^{(1)}+f_{k} h_{i}^{(1)}}{6}+\frac{h_{i, k}^{(2)}}{3}\right) \cdot \nabla_{x y} h_{j}^{(1)} \\
& +K_{G}\left(\frac{f_{j} f_{k}}{6} h_{B}^{(0)}+\frac{f_{j} h_{k}^{(1)}+f_{k} h_{j}^{(1)}}{6}+\frac{h_{j, k}^{(2)}}{3}\right) \cdot \nabla_{x y} h_{i}^{(1)} \\
& +K_{G}\left(\frac{f_{i} h_{B}^{(0)}+h_{i}^{(1)}}{3}\right) \cdot \nabla_{x y} h_{j, k}^{(2)}+K_{G}\left(\frac{f_{j} h_{B}^{(0)}+h_{j}^{(1)}}{3}\right) \\
& +\nabla_{x y} h_{i, k}^{(2)}+K_{G}\left(\frac{f_{k} h_{B}^{(0)}+h_{k}^{(1)}}{3}\right) \nabla_{x y} h_{i, j}^{(2)} \\
& +K_{G}\left(f_{i} \frac{\partial h_{j, k}^{(2)}}{\partial z}+f_{j} \frac{\partial h_{i, k}^{(2)}}{\partial z}+f_{k} \frac{\partial h_{i, j}^{(2)}}{\partial z}\right) / 3 \\
& +K_{G}\left(f_{i} f_{j} \frac{\partial h_{k}^{(1)}}{\partial z}+f_{i} f_{k} \frac{\left.\partial f_{j} f_{k} \frac{\partial h^{(1)}}{\partial z}+f_{j} f_{k} \frac{\partial h_{i}^{(1)}}{\partial z}\right) / 6}{\partial z}\right) \\
& \\
& \\
&
\end{aligned}
$$$$
g_{c_{i, j, k}}^{(3)}=\nabla \cdot\left[K_{G}\left(f_{i} \nabla h_{j, k}^{(2)}+f_{j} \nabla h_{i, k}^{(2)}+f_{k} \nabla h_{i, j}^{(2)}\right) / 3\right.
$$$$
\left.+K_{G}\left(f_{i} f_{j} \nabla h_{k}^{(1)}+f_{i} f_{k} \nabla h_{j}^{(1)}+f_{j} f_{k} \nabla_{i}^{(1)}\right) / 6+K_{G} \frac{f_{i} f_{j} f_{k}}{6} \nabla h^{(0)}\right] .
$$

In general, at $m$ th order and mode $i_{1}, i_{2}, \ldots, i_{m}, m \geq 1$,

$$
\begin{aligned}
\nabla_{x y} \cdot & {\left[K_{G} h_{B}^{(0)} \nabla_{x y} h_{i_{1}, i_{2}, \ldots, i_{m}}^{(m)}\right]+K_{G} \frac{\partial h_{i_{1}, i_{2}, \ldots, i_{m}}^{(m)}}{\partial z}+g_{u_{i_{1}, i_{2}, \ldots, i_{m}}^{(m)}} } \\
& =S_{y} \frac{\partial h_{i_{1}, i_{2}, \ldots, i_{m}}^{(m)}}{\partial t}
\end{aligned}
$$

$$
\nabla \cdot\left[K_{G} \nabla h_{i_{1}, i_{2}, \ldots, i_{m}}^{(m)}\right]+g_{c_{i_{1}, i_{2}, \ldots, i_{m}}^{(m)}}^{(m)}=S_{S} \frac{\partial h_{i_{1}, i_{2}, \ldots, i_{m}}^{(m)}}{\partial t}
$$

subject to initial and boundary conditions,

$$
\begin{array}{ll}
h_{i_{1}, i_{2}, \ldots, i_{m}}^{(m)}(\boldsymbol{x}, 0)=0, & \boldsymbol{x} \in D, \\
h_{i_{1}, i_{2}, \ldots, i_{m}}^{(m)}(\boldsymbol{x}, t)=0, & \boldsymbol{x} \in \Gamma_{D},
\end{array}
$$

$K_{G} \nabla h_{i_{1}, i_{2}, \ldots, i_{m}}^{(m)}(\boldsymbol{x}, t) \cdot \boldsymbol{n}(\boldsymbol{x})=\frac{(-1)^{m+1}}{m !}\left(\prod_{j=1}^{m} f_{i_{j}}\right) \cdot Q(\boldsymbol{x}, t)$,

$x \in \Gamma_{N}$,

where

$$
\begin{aligned}
g_{u_{i_{1}, i_{2}, \ldots, i_{m}}}^{(m)}= & \nabla_{x y} \cdot\left\{K _ { G } \sum _ { k = 1 } ^ { m } \sum _ { i = 0 } ^ { k } \left[\frac{(m-k+i) !}{m !} \sum_{P_{i_{1}, i_{2}, \ldots, i_{m}}}\right.\right. \\
& \left.\left.\cdot \prod_{j=1}^{k-i} f_{i_{j}} h_{i_{k-i+1}, i_{k-i+2, \ldots, i_{k}}}^{(i)} \nabla_{x y} h_{i_{k+1}, \ldots, i_{m}}^{(m-k)}\right]\right\}+K_{G} \\
& \left.\cdot \sum_{k=1}^{m} \frac{(m-k) !}{m !} \sum_{P_{i_{1}, i_{2}, \ldots, i_{m}}} \prod_{j=1}^{k} f_{i_{j}} \frac{\partial h_{i_{k+1}, \ldots, i_{m}}^{(m-k)}}{\partial z}\right], \\
g_{c_{i_{1}, i_{2}, \ldots, i_{m}}^{(m)}=} & \nabla \cdot\left\{K_{G} \sum_{k=1}^{m}\left[\frac{(m-k) !}{m !} \sum_{P_{i_{1}, i_{2}, \ldots, i_{m}}} \cdot \prod_{j=1}^{k} f_{i_{j}} \nabla h_{i_{k+1}, \ldots, i_{m}}^{(m-k)}\right]\right\} .
\end{aligned}
$$

The summation $\sum_{P_{i_{1}, i_{2}, \ldots, i_{m}}}$ in (B20) and (B21) is taken over a subset of the permutation of $\left\{i_{1}, i_{2}, \ldots, i_{m}\right\}$ in which repeated terms of $h_{\left.i_{1}, i_{2}, \cdots\right)}^{(m-k), i_{m-k}}$ are excluded. For example, $\sum_{p_{i}} f_{i} \nabla h_{j, k}^{(2)}=f_{i} \nabla h_{j, k}^{(2)}+f_{j} \nabla h_{i, k}^{(2)}+f_{k} \nabla h_{i, j}^{(2)}$. The term $f_{i} \nabla h_{k, j}^{(2)}$ is identical to $f_{i} \nabla h_{j, k}^{(2)}$ and thereby excluded as $h_{j, k}^{(2)}$ calculated this way is symmetric with respect to its subscript indices.

[49] Acknowledgments. This work is partially supported by Los Alamos National Laboratory through its LDRD/DR project "Science of Geological Carbon Sequestration," by the U.S. National Science Foundation through grant OISE 0511496, by the American Chemical Society through its Petroleum Research Fund (grant 42109-AC9), and by the Chinese Natural Science Foundation through grant 50310444. The authors are grateful to Associate Editor Aldo Fiori and three anonymous reviewers, whose constructive comments have led to an improvement of the manuscript.

\section{References}

Amir, O., and S. P. Neuman (2001), Gaussian closure of one-dimensional unsaturated flow in randomly heterogeneous soils, Transp. Porous Media, 44, 355-383.

Ballio, F., and A. Guadagnini (2004), Convergence assessment of numerical Monte Carlo simulations in groundwater hydrology, Water Resour. Res., 40, W04603, doi:10.1029/2003WR002876.

Bear, J. (1972), Dynamics of Fluids in Porous Media, Dover, Mineola, N. Y. Chen, M., D. Zhang, A. A. Keller, and Z. Lu (2005), A stochastic analysis of steady state two-phase flow in heterogeneous media, Water Resour. Res., 41, W01006, doi:10.1029/2004WR003412. 
Courant, R., and D. Hilbert (1953), Methods of Mathematical Physics, Wiley-Interscience, Hoboken, N. J.

Cushman, J. H. (1997), The Physics of Fluids in Hierarchical Porous Media: Angstroms to Miles, Springer, New York.

Dagan, G. (1982), Stochastic modeling of groundwater flow by unconditional and conditional probabilities: 1. Conditional simulation and the direct problem, Water Resour. Res., 18, 813-833.

Dagan, G. (1985), Stochastic modeling of groundwater flow by unconditional and conditional probabilities: The inverse problem, Water Resour. Res., 21, 65-72.

Dagan, G. (1989), Flow and Transport in Porous Formations, Springer, New York.

Deng, F.-W., and J. H. Cushman (1995), On higher-order corrections to the flow velocity covariance tensor, Water Resour. Res., 31(7), 1659-1672.

Gelhar, L. W. (1993), Stochastic Subsurface Hydrology, Prentice-Hall, Upper Saddle River, N. J.

Ghanem, R. (1998), Scale of fluctuation and the propagation of uncertainty in random porous media, Water Resour. Res., 34, 2123-2136.

Ghanem, R., and S. Dham (1998), Stochastic finite element analysis for multiphase flow in heterogeneous porous media, Transp. Porous Media, $32,239-262$.

Ghanem, R., and D. Spanos (1991), Stochastic Finite Elements: A Spectral Approach, Springer, New York.

Graham, W. D., and D. McLaughlin (1989), Stochastic analysis of nonstationary subsurface solute transport: 1. Unconditional moments, Water Resour. Res., 25, 215-232.

Harbaugh, A. W., E. R. Banta, M. C. Hill, and M. G. McDonald (2000), MODFLOW-2000: The U.S. Geological Survey modular ground-water model-User guide to modularization concepts and the ground-water flow processes, U.S. Geol. Surv. Open File Rep., 00-92, 121 pp.

Hassan, A. E., J. H. Cushman, and J. W. Delleur (1998), A Monte Carlo assessment of Eulerian flow and transport perturbation models, Water Resour. Res., 34, 1143-1163.

Hsu, K. C., D. Zhang, and S. P. Neuman (1996), Higher-order effects on flow and transport in randomly heterogeneous porous media, Water Resour. Res., 32, 571-582.

Loeve, M. (1977), Probability Theory, 4th ed., Springer, New York.

Lu, Z., and D. Zhang (2004a), A comparative study on quantifying uncertainty of flow in heterogeneous media using Monte Carlo simulations, the conventional and the KL-based moment-equation approaches, SIAM J. Sci. Comput., 26(2), 558-577.

Lu, Z., and D. Zhang (2004b), Conditional simulations of flow in randomly heterogeneous porous media using a KL-based moment-equation approach, Adv. Water Resour., 27, 859-874.

Neuman, S. P. (1993), Eulerian-Lagrangian theory of transport in spacetime nonstationary velocity fields: Exact nonlocal formalism by conditional moments and weak approximations, Water Resour. Res., 29, 633646.

Press, W., S. Teukolsky, W. Vetterling, and B. Flannery (1992), Numerical Recipes in $C, 2$ nd ed., Cambridge Univ. Press, New York.

Rubin, Y. (2003), Applied Stochastic Hydrogeology, Oxford Univ. Press, New York.

Yang, J., D. Zhang, and Z. Lu (2004), Stochastic analysis of saturatedunsaturated flow in heterogeneous media by combining Karhunen-Loeve expansion and perturbation method, J. Hydrol., 294, 18-38.

Zhang, D. (1998), Numerical solutions to statistical moment equations of groundwater flow in nonstationary, bounded heterogeneous media, Water Resour. Res., 34, 529-538.

Zhang, D. (2002), Stochastic Methods for Flow in Porous Media: Coping With Uncertainties, 350 pp., Elsevier, New York.

Zhang, D., and Z. Lu (2002), Stochastic analysis of flow in a heterogeneous unsaturated-saturated system, Water Resour. Res., 38(2), 1018, doi:10.1029/2001WR000515.

Zhang, D., and Z. Lu (2004), An efficient, high-order perturbation approach for flow in random porous media via Karhunen-Loéve and polynomial expansions, J. Comput. Phys., 194, 773-794.

G. Liu, Kansas Geological Survey, University of Kansas, Lawrence, KS 66047-3726, USA. (gliu@kgs.ku.edu)

Z. Lu, Hydrology and Geochemistry Group, Los Alamos National Laboratory, Los Alamos, NM 87545, USA.

D. Zhang, Mewbourne School of Petroleum and Geological Engineering, University of Oklahoma, Norman, OK 73019, USA. 